\title{
DENSITY IN SMALL TIME FOR LEVY PROCESSES
}

\author{
JEAN PICARD
}

\begin{abstract}
The density of real-valued Lévy processes is studied in small time under the assumption that the process has many small jumps. We prove that the real line can be divided into three subsets on which the density is smaller and smaller: the set of points that the process can reach with a finite number of jumps ( $\Delta$-accessible points); the set of points that the process can reach with an infinite number of jumps (asymptotically $\Delta$-accessible points); and the set of points that the process cannot reach by jumping ( $\Delta$-inaccessible points).
\end{abstract}

\section{INTRODUCTION}

The problem of the absolute continuity of infinitely divisible laws has been studied for a long time in the literature, especially in dimension 1, see Tucker (1965) (in larger dimension, more geometry is involved, see Yamazato (1994) for an example). Variables corresponding to these laws can be viewed as the values at a fixed time of Lévy processes $X_{t}$ (processes with independent and stationary increments such that $X_{0}=0$ ), or as linear functionals of Poisson random measures. The problem of the absolute continuity and of the smoothness of the density can also be extended to some non linear functionals of Poisson measures, especially to Markov processes $X_{t}$ with jumps (see for instance Bismut (1983), Bichteler et al. (1987), Picard (1996)). In order to study these processes, one has to consider the measure

$$
\mu(y, d x)=\mathbb{P}\left[X_{t+d t} \in(y+d x) \mid X_{t}=y\right] / d t
$$

which describes how the process can jump from $y$ to $y+x$; in the case of Lévy processes, $\mu(y, d x)=\mu(d x)$ does not depend on $y$ and is called the Lévy measure of the process. The sufficient conditions which are known for the existence of a smooth density involve two types of conditions, namely the mass of $\mu$ near 0 (the process must have many small jumps) and the smoothness of $\mu$. Actually, one type of condition can be weakened if the other one is strengthened; for instance, a Lévy process has an absolutely continuous law if its Lévy measure is absolutely continuous and if it has infinitely many jumps (Tucker (1962)), but if the Lévy measure is singular, one has to impose a stronger condition on the number of small jumps; moreover a critical behaviour is possible, where the law is singular for small times and absolutely continuous for large times (Tucker (1965)). If now we assume that the law of $X_{t}$ is absolutely continuous for any $t$, a natural

URL address of the journal: http://www.emath.fr/ps/

Received by the journal November 30, 1995. Revised January 7, 1997. Accepted for publication September 18, 1997.

(c) Société de Mathématiques Appliquées et Industrielles. Typeset by $\mathrm{T}_{\mathrm{EX}}$. 
question is to study the behaviour of the density $p(t, x)$ as $t \rightarrow 0$. This has been considered in Léandre (1987), Ishikawa (1993 and 1994) in the case of absolutely continuous Lévy measures, and for a class of points $x$ which the process can reach with a finite number of jumps (these points will be called $\Delta$-accessible in this paper). Our aim here is to consider the case of processes with possibly singular Lévy measures and with a large enough number of jumps, so that a $C^{\infty}$ density $p(t, x)$ exists for any $t$; however, in order to avoid many technicalities, we consider neither the general case of Markov processes (which requires the use of Malliavin's calculus), nor the multidimensional case (which involves more geometry); we limit ourselves to real-valued Lévy processes and study the logarithmic behaviour of $p(t, x)$ as $t \rightarrow 0$; it appears that this simple case already involves some interesting geometrical properties.

We now state our results without making precise all the conditions. We need two main assumptions on the Lévy process $X_{t}$. Loosely speaking, (a) the first assumption says that $X$ has approximately the same number of small jumps than stable processes with some index $0<\beta \leq 2$; the case $\beta=2$ means that $X$ contains a non trivial Brownian part, and if $\beta<2$, the precise statement of the assumption says that the tail at 0 of the Lévy measure of $X$ satisfies an approximate scaling property;

(b) the second assumption requires that the process goes sufficiently up and down; this assumption is always satisfied if $\beta>1$, and otherwise, it says that the Lévy measure has enough mass on both $\mathbb{R}_{+}^{\star}$ and $\mathbb{R}_{-}^{\star}$.

Under these two assumptions, it appears that the points $x \in \mathbb{R}$ can be divided into three classes for which the behaviour of $p(t, x)$ as $t \rightarrow 0$ is quite different.

(i) The first class is the set $A$ of $\Delta$-accessible points $x$ that the process can reach with a finite number of jumps (in particular $x=0$ that the process can reach without any jump); more precisely, $A$ is the set of points of the form $\sum_{1}^{n} x_{i}$ where $x_{i}$ is in the support of the Lévy measure; for these points (and under additional regularity conditions),

$$
\log p(t, x)=\Gamma(x) \log t+o(\log (1 / t))
$$

where the rate function $\Gamma(x)$ depends on the jumps $x_{i}$ which drive the process from 0 to $x$; in particular, $\Gamma(0)=-1 / \beta$. Notice that this set $A$ may be countable. A large deviation principle is easily proved for the law of $X_{t}$, but the rate function $M(x)$, which is the minimal number of jumps which are necessary to reach $x$, is different from $\Gamma(x)$ in the singular case.

(ii) The second class is the set $\bar{A} \backslash A$ of asymptotically $\Delta$-accessible points that the process can reach with an infinite number of jumps; for these points,

$$
\log (1 / t) \ll \log (1 / p(t, x)) \leq C(\log t)^{2} .
$$

Actually, we will describe an example for which

$$
\log p(t, x) \sim-\rho(x)(\log t)^{2}
$$

for some points $x$ and a function $\rho(x)$ depending on the sequences of jumps driving the process from 0 to $x$. 
(iii) Finally the third class is the set $\mathbb{R} \backslash \bar{A}$ of $\Delta$-inaccessible points that the process cannot reach with only jumps (this does not mean that these points are inaccessible for the process); under our two assumptions $(a)$ and $(b)$, this set can be non empty only if $\beta=2$ ( $X$ has a non trivial Brownian part), or if $1<\beta<2$ and all the jumps of $X$ have the same sign, for instance positive (the process is said to be completely asymmetric, or spectrally positive); in this case $\mathbb{R} \backslash \bar{A}$ is $\mathbb{R}_{-}^{\star}$ and $\log (1 / p(t, x))$ is of order $t^{-1 /(\beta-1)}$.

We also consider the case of non-decreasing Lévy processes (or subordinators). In this case, the second assumption (b) is not satisfied; however, similar properties can also be proved; the main difference concerns the behaviour at $\Delta$-accessible points (notice for instance that $p(t, 0)=0$ under the first assumption (a)).

Let us now set the notations which are used throughout this paper. Consider first an infinitely divisible law $Q$ on $\mathbb{R}$ (or equivalently an infinitely divisible variable $X$ with law $Q$ ); its Fourier transform is given by the LévyKhintchine formula

$\phi(w)=\int e^{i w x} d Q(x)=\exp \left(i w \chi-\frac{\sigma^{2} w^{2}}{2}+\int\left(e^{i w x}-1-i w x 1_{[0,1]}(|x|)\right) d \mu(x)\right)$

with $\chi \in \mathbb{R}, \sigma \geq 0$ (diffusion coefficient) and $\mu$ a measure on $\mathbb{R}^{\star}$ satisfying

$$
\int\left(x^{2} \wedge 1\right) d \mu(x)<\infty
$$

and which is the Lévy measure of $Q$. More generally, if $\lambda=\lambda_{1}+i \lambda_{2}$ is a complex number such that $e^{\lambda_{1} x}$ is $\mu$-integrable on $[-1,1]^{c}$, then

$$
\int e^{\lambda x} d Q(x)=\exp \left(\lambda \chi+\frac{\sigma^{2} \lambda^{2}}{2}+\int\left(e^{\lambda x}-1-\lambda x 1_{[0,1]}(|x|)\right) d \mu(x)\right) .
$$

We also introduce a finite measure on $\mathbb{R}$ which is deduced from $\mu$ and $\sigma$ by

$$
\nu(d x)=\left(x^{2} \wedge 1\right) \mu(d x)+\sigma^{2} \delta_{0}(d x) .
$$

Then $Q$ is characterized by the parameters $(\chi, \sigma, \mu)$, or equivalently by $(\chi, \nu)$ which are called its $\chi$-parameter and $\nu$-measure. It follows easily from (1.1) that the sum of two independent infinitely divisible variables with parameters $\left(\chi_{1}, \nu_{1}\right)$ and $\left(\chi_{2}, \nu_{2}\right)$ is infinitely divisible with parameters $\left(\chi_{1}+\chi_{2}, \nu_{1}+\nu_{2}\right)$; thus, if we decompose the measure $\nu$ into $\nu_{1}+\nu_{2}$, we deduce a decomposition of the variable $X$ into the sum of two independent variables. It is also an easy consequence of (1.1) that if the restriction of $\mu$ to $[-1,1]^{c}$ has a first moment or a second moment, then a variable $X$ with law $Q$ satisfies the same property and

$$
\mathbb{E} X=\chi+\int_{|x|>1} x d \mu(x), \quad \text { var } X=\sigma^{2}+\int x^{2} d \mu(x) .
$$

Now consider a Lévy process $X_{t}$; the law of each variable $X_{t}$ is infinitely divisible, and the parameters $\chi, \sigma, \mu, \nu$ of the process $\left(X_{t}\right)$ are defined to 
be the corresponding parameters of the variable $X_{1}$; then the parameters of the variable $X_{t}$ are given by $t \chi$ and $t \nu$; thus the characteristic function $\phi_{t}$ of $X_{t}$ is deduced from the Lévy-Khintchine formula (1.1), and a decomposition of $\nu$ also leads to a decomposition of $X_{t}$ into the sum of independent Lévy processes. In the particular case where $\sigma=0$ and

$$
\int(|x| \wedge 1) d \mu(x)<\infty
$$

then $X_{t}$ has finite variation and can be written as

$$
X_{t}=\sum_{s \leq t} \Delta X_{s}+t \bar{\chi}
$$

where the $\bar{\chi}$-parameter is defined as

$$
\bar{\chi}=\chi-\int_{[-1,1]} x d \mu(x) .
$$

In this case, the law of $X$ can be characterized by $(\bar{\chi}, \mu)$. In particular, $\bar{\chi}=0$ means that $X_{t}$ is a pure jump process; similarly, we will say that an infinitely divisible law satisfying (1.4) and $\sigma=0$ is of pure jump type if its $\bar{\chi}$-parameter defined by (1.6) is 0 . If $\bar{\chi} \geq 0$ and $\mu$ is supported by $\mathbb{R}_{+}^{*}$, then $X_{t}$ is non decreasing (it is a subordinator).

The paper is organized as follows. In $\S 2$, we prove some preliminary results concerning infinitely divisible variables. In $\S 3$, assuming that the tail of $\nu$ at 0 satisfies an approximate scaling property, we estimate $\sup _{x} p(t, x)$. In $\S 4$, we study $p(t, 0)$ and in the three subsequent sections, we study $p(t, x)$ when $x$ is $\Delta$-accessible ( $\S 5$ ), asymptotically $\Delta$-accessible $(\S 6)$, or $\Delta$-inaccessible $(\S 7)$; in $\S 5$, we also give the large deviation principle. The various constant numbers will be denoted by $c$ or $C$ and may vary from an equation to the other.

\section{Preliminary Results}

LEMMA 2.1. Let $Q_{i}$ be a family of infinitely divisible laws with parameters $\chi_{i}$ and $\nu_{i}$. Suppose that the total mass $\nu_{i}(\mathbb{R})$ is bounded, and that each $Q_{i}$ has a continuous density $p_{i}$. Then there exists a family $x_{i}$ such that $x_{i}-\chi_{i}$ is bounded and $p_{i}\left(x_{i}\right)$ is bounded below by a positive number. In particular, $\sup _{x} p_{i}(x)$ is bounded below.

Proof. Let $X_{i}$ be a variable with law $Q_{i}$, and write $X_{i}=\chi_{i}+Y_{i}+Z_{i}$ where $Y_{i}$ and $Z_{i}$ are independent infinitely divisible variables with $\chi$-parameter 0 , and the $\nu$-measures of which are respectively the restriction of $\nu_{i}$ to $[-1,1]$ and its complement; notice that $Z_{i}$ has $\bar{\chi}$-parameter 0 (see (1.6)). Then

$$
\mathbb{E} Y_{i}^{2}=\nu_{i}([-1,1]), \quad \mathbb{P}\left[Z_{i}=0\right] \geq \exp -\mu_{i}\left([-1,1]^{c}\right)=\exp -\nu_{i}\left([-1,1]^{c}\right)
$$

where the second relation is obtained by considering $Z_{i}$ as the value at time 1 of a pure jump Lévy process, and by noticing that the right-hand side is 
the probability for the process to contain no jump. Thus

$$
\begin{aligned}
\mathbb{P}\left[\left|X_{i}-\chi_{i}\right| \leq h\right] & \geq \mathbb{P}\left[\left|Y_{i}\right| \leq h\right] \mathbb{P}\left[Z_{i}=0\right] \\
& \geq\left(1-\nu_{i}([-1,1]) / h^{2}\right) \exp -\nu_{i}\left([-1,1]^{c}\right) \\
& \geq\left(1-\nu_{i}(\mathbb{R}) / h^{2}\right) \exp -\nu_{i}(\mathbb{R})
\end{aligned}
$$

if we choose $h$ large enough; this expression can be bounded below by a positive constant, so the supremum of $p_{i}$ on $\left[\chi_{i}-h, \chi_{i}+h\right]$ can also be bounded below.

Lemma 2.2. Consider a family $Q_{i}$ of infinitely divisible laws of pure jump type, with Lévy measure $\mu_{i}$ satisfying

$$
\lim _{\varepsilon \rightarrow 0} \sup _{i} \int_{[-\varepsilon, \varepsilon]}|x| d \mu_{i}(x)=0, \quad \sup _{i} \nu_{i}(\mathbb{R})<\infty .
$$

Suppose that each $Q_{i}$ has a continuous density $p_{i}$. Then for any fixed $h>0$, there exists a family $x_{i}$ such that $\left|x_{i}\right| \leq h$ and $p_{i}\left(x_{i}\right)$ is bounded below by a positive number.

Proof. Let $0<\varepsilon \leq 1$ be a number which will be chosen later. We decompose a variable with law $Q_{i}$ into $X_{i}=Y_{i}+Z_{i}$, where $Y_{i}$ and $Z_{i}$ are of pure jump type, and their Lévy measures are the restriction of $\mu_{i}$ to $[-\varepsilon, \varepsilon]$ and its complement. Then

$$
\begin{aligned}
\mathbb{E} Y_{i}^{2} & =\left(\int_{[-\varepsilon, \varepsilon]} x d \mu_{i}(x)\right)^{2}+\int_{[-\varepsilon, \varepsilon]} x^{2} d \mu_{i}(x) \\
& \leq\left(\int_{[-\varepsilon, \varepsilon]} x d \mu_{i}(x)\right)^{2}+\varepsilon \int_{[-\varepsilon, \varepsilon]}|x| d \mu_{i}(x)
\end{aligned}
$$

converges uniformly to 0 as $\varepsilon \rightarrow 0$, so we can choose $\varepsilon$ such that $\mathbb{E} Y_{i}^{2} \leq h^{2} / 2$. On the other hand,

$$
\mathbb{P}\left[Z_{i}=0\right] \geq \exp -\mu_{i}\left([-\varepsilon, \varepsilon]^{c}\right) \geq \exp -\nu_{i}(\mathbb{R}) / \varepsilon^{2}
$$

so by proceeding as in Lemma 2.1,

$$
\mathbb{P}\left[\left|X_{i}\right| \leq h\right] \geq\left(1-\mathbb{E} Y_{i}^{2} / h^{2}\right) \exp -\nu_{i}(\mathbb{R}) / \varepsilon^{2} \geq\left(\exp -\nu_{i}(\mathbb{R}) / \varepsilon^{2}\right) / 2
$$

is bounded below and we can conclude.

Lemma 2.3. Consider a family of infinitely divisible laws $Q_{i}$ with parameters $\left(\chi_{i}, \nu_{i}\right)$ and suppose that

$$
\nu_{i}([-\varepsilon, \varepsilon]) \geq c \varepsilon^{2-\beta}
$$

for any $0 \leq \varepsilon \leq 1$ and for some $c>0$ and $0<\beta \leq 2$. Then $Q_{i}$ has a smooth density $p_{i}$, and $p_{i}(x)$, as well as all the derivatives $p_{i}^{(k)}(x)$, are bounded uniformly in $(i, x)$.

REMARK 2.4. The proof relies on integrability properties of the characteristic function, and is classical, see $\S 4 \mathrm{f}$ of Bismut (1983) for related results; 
actually, it can be extended to some Markov processes with jumps, see Picard (1996).

Proof. We deduce from the Lévy-Khintchine formula (1.1) that the characteristic function of $Q_{j}$ satisfies

$$
\begin{aligned}
\left|\phi_{j}(w)\right| & =\exp \left(-\int(1-\cos (w x)) d \mu_{j}(x)-\sigma_{j}^{2} w^{2} / 2\right) \\
& \leq \exp \left(-c_{0} w^{2} \nu_{j}([-1 / w, 1 / w])\right)
\end{aligned}
$$

for $|w| \geq 1$ and some $c_{0}>0$, where we have used the inequality

$$
1-\cos (w x) \geq c_{0}|w x|^{2}
$$

on the set $\{x ;|w x| \leq 1\}$. Thus, from our assumption (2.1),

$$
\left|\phi_{j}(w)\right| \leq \exp -c|w|^{\beta}
$$

for $|w| \geq 1$. In particular, $\phi_{j}$ is integrable with respect to the Lebesgue measure, so $Q_{j}$ has a density $p_{j}$ which is given by the inversion formula

$$
p_{j}(x)=\frac{1}{2 \pi} \int e^{-i w x} \phi_{j}(w) d w \leq \frac{1}{2 \pi} \int\left|\phi_{j}(w)\right| d w .
$$

Thus $p_{j}$ is uniformly bounded. Similarly, by differentiating this formula, one proves that

$$
\sup _{x}\left|p_{j}^{(k)}(x)\right| \leq \frac{1}{2 \pi} \int|w|^{k}\left|\phi_{j}(w)\right| d w
$$

is bounded.

The following lemma is a simple result concerning the closed support of infinitely divisible laws. In particular, it does not say anything about much more complicated problems (such as the Hausdorff dimension of the law, see Rubin (1967)) which have been studied in previous literature; it is however sufficient for our purpose, since our laws will be easily proved to have a smooth density from previous lemma.

Lemma 2.5. Consider an infinitely divisible law $Q$ without Gaussian part $(\sigma=0)$ and the Lévy measure of which satisfies $\mu([-\varepsilon, \varepsilon])>0$ for any $\varepsilon>0$. Suppose either that

$$
\mu(]-\infty, 0[) \mu(] 0,+\infty[)>0,
$$

or that

$$
\int(|x| \wedge 1) d \mu(x)=+\infty
$$

Then the support of $Q$ is $\mathbb{R}$. If these two conditions are not satisfied, then the support is $]-\infty, \bar{\chi}]$ or $[\bar{\chi},+\infty[$.

REMARK 2.6. It is evident that the support is $\mathbb{R}$ if $Q$ has a non trivial Gaussian part.

Proof. For any $\varepsilon>0$, if $\mu_{\varepsilon}$ is the restriction of $\mu$ to $[-\varepsilon, \varepsilon]^{c}$, one can check that $Q * \mu_{\varepsilon}$ is absolutely continuous with respect to $Q$. One deduces that

$$
\operatorname{supp} Q+\operatorname{supp} \mu \subset \operatorname{supp} Q .
$$


From our assumption, the support of $\mu$ contains numbers with arbitrarily small absolute value; suppose for instance that it contains arbitrarily small positive numbers; then we deduce from (2.4) that

$$
x \in \operatorname{supp} Q \Longrightarrow[x,+\infty[\subset \operatorname{supp} Q
$$

It is thus sufficient to prove that under each of our two assumptions (2.2) and (2.3), the support of $Q$ contains arbitrarily large negative numbers, and that if neither (2.2) nor (2.3) hold, the smallest number in the support is $\bar{\chi}$. Under (2.2), the support of $\mu$ contains some negative number $-y$, so (2.4) implies

$$
x \in \operatorname{supp} Q \Longrightarrow(x-y) \in \operatorname{supp} Q
$$

and we conclude. Let us now suppose that (2.2) does not hold, so that $\mu$ is supported by positive numbers. If we view $Q$ as the law of $X_{1}$ for some Lévy process $X_{t}$, then for any $\varepsilon$, the law of

$$
X_{1}^{\varepsilon}=X_{1}-\sum_{t \leq 1} \Delta X_{t} 1_{\left\{\Delta X_{t}>\varepsilon\right\}}
$$

is absolutely continous with respect to $Q$. If (2.3) holds, then $X_{1}^{\varepsilon} \downarrow-\infty$ as $\varepsilon \downarrow 0$, so its support contains some $x(\varepsilon)$ which diverges to $-\infty$, and therefore $\operatorname{supp} Q$ also contains $x(\varepsilon)$; if (2.3) does not hold, then $X_{1}^{c} \downarrow \bar{\chi}$ from (1.5), and we can deduce that $\bar{\chi}$ is in the support of $Q$; moreover in this case, $X_{t}-t \bar{\chi}$ is non-decreasing, so $X_{1} \geq \bar{\chi}$ and the support cannot contain a smaller number.

LeMma 2.7. Let $X$ be an infinitely divisible variable with parameters $(\chi, \nu)$ such that $\nu$ is supported by a bounded interval $[-\rho, \rho]$. There exists a $C>0$ depending only on upper bounds on $|\chi|, \nu(\mathbb{R})$ and $\rho$ such that for any $h$,

$$
\mathbb{P}[|X| \geq h] \leq C e^{-h} .
$$

Remark 2.8. Equivalently, one can say from (1.3) that $C$ depends only on upper bounds on $\rho,|\mathbb{E} X|$ and var $X$.

Proof. It is sufficient to estimate the expectation of $\exp X$ and $\exp -X$. But from (1.2),

$$
\mathbb{E} \exp X=\exp \left(\chi+\frac{\sigma^{2}}{2}+\int\left(e^{x}-1-x 1_{\{|x| \leq 1\}}\right) d \mu(x)\right) .
$$

Since the support of $\mu$ is bounded, the function in the integral is dominated by $x^{2} \wedge 1$, so the term in the exponential is dominated by $|\chi|+\nu(\mathbb{R})$. The expectation of $\exp -X$ is dealt with similarly.

Lemma 2.9. Consider infinitely divisible variables $X$, the Lévy measures of which are supported by some bounded interval $[-\rho, \rho]$, and which have zero mean. Then there exists $C_{n}=C_{n}(\rho)$ such that

$$
\mathbb{P}[|X| \geq n \rho] \leq C_{n}(\operatorname{var} X)^{n}
$$


REMARK 2.10. One can say equivalently that

$$
\mathbb{P}[|X| \geq n \rho] \leq C_{n}^{\prime} \nu(\mathbb{R})^{n}
$$

Proof. Put $\varepsilon=\operatorname{var} X$ and $r=r(\varepsilon)=\varepsilon^{1 /(2(n+1))}$; one can suppose $\varepsilon \leq 1$. Consider $X$ as the value $X_{1}$ of a Lévy process $X_{t}$ at time 1 , and decompose it into $X_{t}=X_{t}^{1}+X_{t}^{2}$ where

$$
X_{t}^{1}=\sum_{s \leq t} 1_{\left\{\left|\Delta X_{s}\right|>r\right\}} \Delta X_{s}
$$

Let us consider the event $\left\{\left|X_{1}^{1}\right| \geq(n-1 / 2) \rho\right\}$; the process $X_{t}^{1}$ is a pure jump process, and its jumps are at most $\rho$ (in absolute value), so on this event, there is at least $n$ jumps; moreover, one more jump is needed if one of the jumps is less than $\rho / 2$. Thus, in order for $\left|X_{1}^{1}\right|$ to be greater than $(n-1 / 2) \rho$, there must be at least $n$ jumps satisfying $\rho / 2 \leq|\Delta X| \leq \rho$, or at least $n+1$ jumps satisfying $r \leq|\Delta X| \leq \rho$. These two numbers of jumps are Poisson variables with mean values

$$
\mu\{|x| \geq \rho / 2\} \leq 4 \varepsilon / \rho^{2}, \quad \mu\{|x| \geq r\} \leq \varepsilon / r^{2}=\varepsilon^{n /(n+1)},
$$

so

$$
\mathbb{P}\left[\left|X_{1}^{1}\right| \geq(n-1 / 2) \rho\right] \leq \mu\{|x| \geq \rho / 2\}^{n}+\mu\{|x| \geq r\}^{n+1}=O\left(\varepsilon^{n}\right) .
$$

The Lévy measure of $X^{2} / r$ is supported by $[-1,1]$,

$$
\left|\mathbb{E} \frac{X_{1}^{2}}{r}\right|=\left|\mathbb{E} \frac{X_{1}^{1}}{r}\right|=\left|\int_{|x|>r} \frac{x}{r} d \mu(x)\right| \leq \varepsilon / r^{2} \leq 1, \quad \text { var } \frac{X_{1}^{2}}{r} \leq \varepsilon / r^{2} \leq 1,
$$

so from Lemma 2.7

$$
\mathbb{P}\left[\left|X_{1}^{2}\right| \geq \rho / 2\right] \leq C e^{-\rho /(2 r)}=o\left(\varepsilon^{k}\right)
$$

for any $k$. We conclude by adding (2.5) and (2.6).

\section{Estimation of THE SUPREMUM OF THE DENSity}

We have seen in $\S 2$ that the supremum of the density of an infinitely divisible variable can be estimated from the behaviour of its $\nu$-measure. We now translate these results in the case of a Lévy process in small time.

Theorem 3.1. Let $X_{t}$ be a Lévy process with parameters $(\chi, \nu)$. (a) Suppose that

$$
\liminf _{\varepsilon \rightarrow 0} \varepsilon^{\beta-2} \nu([-\varepsilon, \varepsilon])>0
$$

for some $0<\beta \leq 2$. Then $X_{t}$ has a $C^{\infty}$ density satisfying

$$
\sup _{x} p(t, x)=O\left(t^{-1 / \beta}\right)
$$


as $t \rightarrow 0$, and more generally

$$
\sup _{x}\left|p^{(k)}(t, x)\right|=O\left(t^{-(k+1) / \beta}\right) .
$$

(b) Suppose that $X_{t}$ has a continuous density $p(t,$.$) and that$

$$
\limsup _{\varepsilon \rightarrow 0} \varepsilon^{\beta-2} \nu([-\varepsilon, \varepsilon])<\infty
$$

for some $0<\beta \leq 2$. Then, for $t$ small enough,

$$
\sup _{x} p(t, x) \geq c t^{-1 / \beta} .
$$

Proof. Consider $Y_{t}=X_{t} / t^{1 / \beta}$. Of course, $Y_{t}$ is not a Lévy process but is for each $t$ an infinitely divisible variable. Its Lévy measure and diffusion coefficient are given by

$$
\mu_{t}(A)=t \mu\left(t^{1 / \beta} A\right), \quad \sigma_{t}=\sigma t^{1 / 2-1 / \beta},
$$

so its $\nu$-measure satisfies

$$
\nu_{t}(\mathbb{R})=t \int\left(\frac{x^{2}}{t^{2 / \beta}} \wedge 1\right) d \mu(x)+t \frac{\sigma^{2}}{t^{2 / \beta}}=t \int \frac{d \nu(x)}{\left(x^{2} \wedge 1\right) \vee t^{2 / \beta}}
$$

if $t \leq 1$, and

$$
\nu_{t}([-\varepsilon, \varepsilon])=t^{1-2 / \beta} \nu\left(\left[-\varepsilon t^{1 / \beta}, \varepsilon t^{1 / \beta}\right]\right)
$$

for $\varepsilon \leq 1$. In case (a), we deduce that

$$
\nu_{t}([-\varepsilon, \varepsilon]) \geq c \varepsilon^{2-\beta},
$$

so from Lemma 2.3, the variables $Y_{t}$ have uniformly bounded densities $q(t,$. with bounded derivatives; thus $X_{t}$ has a density given by

$$
p(t, x)=t^{-1 / \beta} q\left(t, t^{-1 / \beta} x\right)
$$

and we can conclude. In case $(b)$, from an integration by parts,

$$
\nu_{t}(\mathbb{R})=t \nu(\mathbb{R})+2 t \int_{t^{1 / \beta}}^{1} \frac{\nu([-x, x])}{x^{3}} d x
$$

which is bounded from our assumption. Thus we can apply Lemma 2.1 and deduce that the supremum of the density of $Y_{t}$ is bounded below. We again conclude from the formula (3.5) relating the densities of $X_{t}$ and $Y_{t}$.

REMARK 3.2. The assumption (3.2) of case (b) is always satisfied with $\beta=2$, so the Wiener process provides a lower bound for the concentration of Lévy processes in small time.

REMARK 3.3. If $X_{t}$ is a $\beta$-stable process, then $\nu$ satisfies the scaling property

$$
\nu([-\varepsilon, \varepsilon])=\varepsilon^{2-\beta} \nu([-1,1]),
$$


and both conditions ( $a$ ) and (b) hold. These conditions can actually be viewed as an approximate scaling property on the tail of $\nu$ at 0 .

REMARK 3.4. If we assume condition (3.2) with $\beta<1$, then

$$
\int_{[-1,1]}|x| d \mu(x)=\int_{[-1,1]} d \nu(x) /|x|=\nu([-1,1])+\int_{0}^{1} \nu([-x, x]) / x^{2} d x<\infty
$$

so $X_{t}$ has finite variation. If we assume condition (3.1) with $\beta \geq 1$, then

$$
\int_{[-\varepsilon, \varepsilon]}|x| d \mu(x)=\int_{[-\varepsilon, \varepsilon]} d \nu(x) /|x| \geq \nu([-\varepsilon, \varepsilon]) / \varepsilon \geq c \varepsilon^{1-\beta} \geq c,
$$

so

$$
\int_{[-1,1]}|x| d \mu(x)=\infty
$$

and $X_{t}$ has infinite variation.

REMARK 3.5. By joining the two parts of Theorem 3.1, we deduce that

$$
c \varepsilon^{2-\beta} \leq \nu([-\varepsilon, \varepsilon]) \leq C \varepsilon^{2-\beta} \Longrightarrow c^{\prime} t^{-1 / \beta} \leq \sup _{x} p(t, x) \leq C^{\prime} t^{-1 / \beta},
$$

and that

$$
\lim _{\varepsilon \rightarrow 0}(\log \nu([-\varepsilon, \varepsilon]) / \log \varepsilon)=2-\beta
$$

implies

$$
\lim _{t \rightarrow 0}(\sup \log p(t, x) / \log t)=-1 / \beta
$$

\section{ESTIMATION NEAR 0}

We now want to find a condition ensuring that $p(t, 0)$ also satisfies a lower bound similar to (3.3), so that it has the order of magnitude of $\sup _{x} p(t, x)$. Consider first the symmetric case $\chi=0$ and $\mu$ symmetric; in this case, the function $x \mapsto p(t, x)$ has its maximum at $x=0$ (because the characteristic function is positive), and therefore the behaviour of $p(t, 0)$ follows from Theorem 3.1. Let us now consider the general non-symmetric case.

LEMMA 4.1. Let $Q_{i}$ be a family of infinitely divisible laws with parameters $\left(\chi_{i}, \nu_{i}\right)$, and let $0<\beta \leq 2$. Suppose that $\chi_{i}$ and $\nu_{i}(\mathbb{R})$ are uniformly bounded and that one of the two following conditions is satisfied;

(a) $\beta \geq 1$ and $\nu_{i}$ satisfies the lower bound condition (2.1);

(b) $\beta<1$ and both restrictions of $\nu_{i}$ to $\mathbb{R}_{+}$and $\mathbb{R}_{-}$satisfy (2.1).

Then for any $\rho>0$, the density $p_{i}$ of $Q_{i}$ (which exists from Lemma 2.3) satisfies

$$
\inf _{i} \inf _{|x| \leq \rho} p_{i}(x)>0 .
$$

Proof. Let $\nu_{i}^{1}$ be the restriction of $\nu_{i} / 2$ to $[-1,1]$, let $\nu_{i}^{2}=\nu_{i}-\nu_{i}^{1}$, and decompose a variable $X_{i}$ with law $Q_{i}$ into independent variables $X_{i}^{1}+X_{i}^{2}$ with respective coefficients $\left(\nu_{i}^{1}, 0\right)$ and $\left(\nu_{i}^{2}, \chi_{i}\right)$. Let $p_{i}^{2}$ be the density of $X_{i}^{2}$; 
the mass $\nu_{i}^{2}(\mathbb{R})$ and the coefficient $\chi_{i}$ are bounded, so, from Lemma 2.1, there exists a bounded $x_{i}$ such that $p_{i}^{2}\left(x_{i}\right)$ is bounded below by a positive number; moreover, the derivative of $p_{i}^{2}$ is uniformly bounded from Lemma 2.3 , so there exists a positive $c$ such that

$$
\forall x \in \mathbb{R} \quad\left|x-x_{i}\right| \leq c \Longrightarrow p_{i}^{2}(x) \geq c .
$$

If we notice that $p_{i}(x)$ is the expectation of $p_{i}^{2}\left(x-X_{i}^{1}\right)$, we obtain

$$
\begin{aligned}
\inf _{|x| \leq \rho} p_{i}(x) & \geq c \inf _{|x| \leq \rho} \mathbb{P}\left[\left|x-X_{i}^{1}-x_{i}\right| \leq c\right] \\
& \geq c \inf _{|x| \leq \rho+C} \mathbb{P}\left[\left|X_{i}^{1}-x\right| \leq c\right] .
\end{aligned}
$$

Thus it is sufficient to check that for any $C$ and $c$,

$$
\inf _{i} \inf _{|x| \leq C} \mathbb{P}\left[\left|X_{i}^{1}-x\right|<c\right]>0
$$

or that

$$
\inf _{X \in \mathcal{X}} \inf _{|x| \leq C} \mathbb{P}[|X-x|<c]>0
$$

where $\mathcal{X}$ denotes the set of laws of $X_{i}^{1}$. The measures $\nu_{i}^{1}$ are relatively compact for the topology of convergence on bounded functions (they are bounded and supported by $[-1,1]$ ), and for any converging subsequence, the corresponding $X_{i}^{1}$ also converges in law (this is an easy application of the Lévy-Khintchine formula (1.1)); thus $\mathcal{X}$ is relatively compact. Since the map $(x, X) \mapsto \mathbb{P}[|X-x|<c]$ is lower semicontinuous, it is sufficient to prove that

$$
\mathbb{P}[|X-x|<c]>0
$$

for any $x, c$ and any $X$ in the closure $\overline{\mathcal{X}}$ of $\mathcal{X}$. This means that one has to prove that the closed support of any $X \in \overline{\mathcal{X}}$ is $\mathbb{R}$; the $\nu$-measure $\nu^{\prime}$ of $X$ satisfies an estimate of type

$$
\nu^{\prime}([-\varepsilon, \varepsilon]) \geq c \varepsilon^{2-\beta}
$$

because these estimates hold uniformly for $\nu_{i}^{1}$; if $\beta=2, X$ has a non trivial Gaussian part, so the result is immediate; if $1 \leq \beta<2$ and if $\mu^{\prime}$ is the Lévy measure of $X$, the function $|x| \wedge 1$ is not $\mu^{\prime}$-integrable from (3.7), so the result follows from Lemma 2.5; in the case $\beta<1$, both $\nu^{\prime}([-\varepsilon, 0[)$ and $\left.\left.\nu^{\prime}(] 0, \varepsilon\right]\right)$ satisfy the above estimate, so one can also apply Lemma 2.5 .

LEMMA 4.2. Let $Q_{i}$ be a family of infinitely divisible laws of pure jump type; we suppose that the $\nu$-measures $\nu_{i}$ are uniformly bounded, supported by $\mathbb{R}_{+}^{+}$, and that

$$
c \varepsilon^{2-\beta} \leq \nu_{i}([0, \varepsilon]) \leq C \varepsilon^{2-\beta}
$$

for some $0<\beta<1$. Then for any $0<\rho_{1}<\rho_{2}$, the densities $p_{i}$ of $Q_{i}$ satisfy

$$
\inf _{i} \inf _{\rho_{1} \leq x \leq \rho_{2}} p_{i}(x)>0
$$


Proof. We follow the argument of the proof of Lemma 4.1. We decompose $X_{i}$ into $X_{i}^{1}+X_{i}^{2}$ of pure jump type, with $\nu$-measures $\nu_{i}^{1}$ and $\nu_{i}^{2}$ defined as in Lemma 4.1. Notice that

$$
\int_{[0, \varepsilon]} \frac{d \nu_{i}^{2}(y)}{y}=\frac{\nu_{i}^{2}([0, \varepsilon])}{\varepsilon}+\int_{0}^{\varepsilon} \frac{\nu_{i}^{2}([0, y])}{y^{2}} d y \leq C \varepsilon^{1-\beta},
$$

so Lemma 2.2 and the fact that $X_{i}^{2} \geq 0$ show that there exists $0<x_{i}<\rho_{1}$ such that $p_{i}^{2}\left(x_{i}\right) \geq c$. From the boundedness of the derivative of $p_{i}^{2}$, we also deduce

$$
\left|x-x_{i}\right| \leq c \Longrightarrow p_{i}^{2}(x) \geq c .
$$

Thus

$$
\begin{aligned}
\inf _{\rho_{1} \leq x \leq \rho_{2}} p(x) & \geq c \inf _{\rho_{1} \leq x \leq \rho_{2}} \mathbb{P}\left[\left|x-X_{i}^{1}-x_{i}\right| \leq c\right] \\
& \geq c \inf _{0 \leq x \leq \rho_{2}} \mathbb{P}\left[\left|X_{i}^{1}-x\right| \leq c\right] .
\end{aligned}
$$

Therefore one only has to prove that

$$
\inf _{X \in \mathcal{X}} \inf _{0 \leq x \leq \rho_{2}} \mathbb{P}[|X-x|<c]>0
$$

or that the support of any $X \in \overline{\mathcal{X}}$ contains $\mathbb{R}_{+}$; but this follows from Lemma 2.5 , because $X$ is of pure jump type.

Theorem 4.3. Assume that

$$
c \varepsilon^{2-\beta} \leq \nu([-\varepsilon, \varepsilon]) \leq C \varepsilon^{2-\beta}
$$

for any $0 \leq \varepsilon \leq 1$ and some index $0<\beta \leq 2$. Suppose also that one of the four following conditions is satisfied;

(a) $\beta>1$;

(b) $\beta=1$ and

$$
\limsup _{\varepsilon \rightarrow 0}\left|\int_{\{\varepsilon \leq|x| \leq 1\}} x d \mu(x)\right|<\infty ;
$$

(c) $\beta<1$, both restrictions of $\nu$ to $\mathbb{R}_{-}^{*}$ and $\mathbb{R}_{+}^{+}$satisfy (4.1), and the $\bar{\chi}-$ parameter is 0 (so that $X_{t}$ is a pure jump process);

(d) $\beta<1, \nu$ is supported by $\mathbb{R}_{+}^{+}$and the $\bar{\chi}$-parameter is 0 (so that $X_{t}$ is a pure jump non decreasing process).

Then, in cases (abc), for any positive $\rho$, one has

$$
|x| \leq \rho t^{1 / \beta} \Longrightarrow c t^{-1 / \beta} \leq p(t, x) \leq C t^{-1 / \beta}
$$

for t small enough, so in particular $p(t, 0)$ is of order $t^{-1 / \beta}$. In case (d), for any positive $\rho_{1}$ and $\rho_{2}$ and for small $t$,

$$
\rho_{1} t^{1 / \beta} \leq x \leq \rho_{2} t^{1 / \beta} \Longrightarrow c t^{-1 / \beta} \leq p(t, x) \leq C t^{-1 / \beta} .
$$

REMARK 4.4. The conclusion of case $(d)$ is weaker. This is not surprising since $p(t, 0)=0$. One can say in this case that $X$ slips on the right, and one can refer to the cases $(a b c)$ as the non slipping case. 
Proof. In this theorem, the upper bound has already been derived in Theorem 3.1, and for the lower bound, it is sufficient to verify that the assumptions of Lemmas 4.1 or 4.2 are satisfied for the family of variables $Y_{t}=X_{t} / t^{1 / \beta}$. So denote by $\left(\nu_{t}, \chi_{t}\right)$ the coefficients of $Y_{t}$. We have already checked in (3.6) that $\nu_{t}(\mathbb{R})$ is bounded and from (3.4), it is easy to see that $\nu_{t}([-\varepsilon, \varepsilon])$ satisfies the lower and upper bound conditions (on both sides in case $(c))$. Moreover, in case $(d)$, the $\bar{\chi}$-parameter is 0 . Thus we only have to prove that $\chi_{t}$ is bounded in cases $(a),(b)$ and $(c)$. From $(1.3)$, one has

$$
\chi=t^{-1} \mathbb{E}\left[X_{t}-\sum_{s \leq t} \Delta X_{s} 1_{\left\{\Delta X_{s}>1\right\}}\right],
$$

so one can verify that

$$
\begin{aligned}
\chi_{t} & =t^{-1 / \beta} \mathbb{E}\left[X_{t}-\sum_{s \leq t} \Delta X_{s} 1_{\left\{\Delta X_{s}>t^{1 / \beta}\right\}}\right] \\
& =t^{1-1 / \beta}\left(\chi-\int_{\left\{t^{1 / \beta}<|x| \leq 1\right\}} x d \mu(x)\right) .
\end{aligned}
$$

In case (a), one has

$$
\begin{aligned}
\int_{\left\{t^{1 / \beta}<|x| \leq 1\right\}}|x| d \mu(x) \\
=\nu([-1,1])-t^{-1 / \beta} \nu\left(\left[-t^{1 / \beta}, t^{1 / \beta}\right]\right)+\int_{t^{1 / \beta}}^{1} \frac{\nu([-x, x])}{x^{2}} d x \\
=O\left(t^{1 / \beta-1}\right)
\end{aligned}
$$

so $\chi_{t}$ is bounded; it is also immediately proved to be bounded in case $(b)$; in case $(c)$, since $\bar{\chi}=0$, from (1.6) one has

$$
\chi_{t}=t^{1-1 / \beta} \int_{\left\{|x| \leq t^{1 / \beta}\right\}} x d \mu(x)
$$

and

$$
\begin{aligned}
\int_{\left\{|x| \leq t^{1 / \beta}\right\}}|x| d \mu(x) & =t^{-1 / \beta} \nu\left(\left[-t^{1 / \beta}, t^{1 / \beta}\right]\right)+\int_{0}^{t^{1 / \beta}} \frac{\nu([-x, x])}{x^{2}} d x \\
& =O\left(t^{1 / \beta-1}\right) .
\end{aligned}
$$

Thus $\chi_{t}$ is bounded in each of the three cases.

Example 4.5. If $X_{t}$ is a symmetric stable process, it is clear that the conditions of Theorem 4.3, cases (a), (b) or (c), are fulfilled. If now $\sigma=0$ and $\mu$ is supported by $\mathbb{R}_{+}^{+}$with $\mu(d x)=d x / x^{1+\beta}$, we are in case $(a)$ if $1<\beta<2$, and in case (d) if $0<\beta<1$ and $\bar{\chi}=0$. Actually, it appears that if $\beta<1$ and $\bar{\chi}=0$, or if $\beta>1$ and

$$
\chi=-\int_{1}^{\infty} x d \mu(x)=-1 /(\beta-1)
$$


(so that $X_{t}$ is a martingale), then $X_{t}$ satisfies the scaling property

$$
\left(X_{\lambda t} ; t \geq 0\right) \sim\left(\lambda^{1 / \beta} X_{t} ; t \geq 0\right)
$$

for any $\lambda>0$, so that $X_{t}$ is a completely asymmetric stable process (a stable subordinator in the non decreasing case). The case $\beta=1$ has not been studied in the theorem, and no scaling property is possible; by looking more precisely at the proof, it appears that the density can be bounded below at

$$
x=x(t)=-t \int_{t}^{1} x d \mu(x)=-t \log (1 / t) .
$$

This is the only value of $\beta$ for which the slipping of the process is larger than the dispersion of the law for any value of $\chi$.

Example 4.6. Suppose that $\sigma=0$ and $\mu$ is given by

$$
\mu=\sum_{n \in \mathbb{Z}} 2^{n \beta} \delta_{2-n}
$$

for $0<\beta<2$, where $\delta_{y}$ denotes the Dirac mass at $y$. This measure can be viewed as the discretization at points $2^{-n}$ of the previous example. Then

$$
(\varepsilon / 2)^{2-\beta} /\left(1-2^{\beta-2}\right) \leq \nu([0, \varepsilon])=\sum_{n: 2^{n} \geq 1 / \varepsilon} 2^{-n(2-\beta)} \leq \varepsilon^{2-\beta} /\left(1-2^{\beta-2}\right),
$$

so $\nu$ satisfies the approximate scaling property (4.1); if $\beta>1$, we are in the case (a) of Theorem 4.3, and if $\beta<1$ and $\bar{\chi}=0$ (non decreasing process), we are in the case $(d)$. Notice that like previously, if $\beta<1$ and $\bar{\chi}=0$, or if $\beta>1$ and

$$
\chi=-\int_{1}^{\infty} x d \mu(x)=-1 /\left(2^{\beta-1}-1\right),
$$

then $X_{t}$ satisfies the scaling property

$$
\left(X_{2 t} ; t \geq 0\right) \sim\left(2^{1 / \beta} X_{t} ; t \geq 0\right) .
$$

Subsequently, we will refer to (4.2) as the dyadic example.

In Theorem 4.3, one assumes that lower and upper bounds on the tail of $\nu$ at 0 have exactly the same order of magnitude. This was not necessary in $\delta 3$; the logarithmic estimation of $\sup _{x} p(t, x)$ may follow from the logarithmic tail estimation (3.8). Here, one can similarly wonder if

$$
\lim \frac{\log p(t, 0)}{\log t}=-1 / \beta
$$

can follow from (3.8) instead of (4.1). This is of course true in the symmetric case. In other cases however, $p(t, 0)$ may have a much more irregular behaviour; suppose for instance that $\sigma=\chi=0$ and that $\mu$ is supported by a sequence $\left(u_{n}\right)_{n \in \mathbb{N}}$ such that

$$
u_{n+1}=u_{n} /\left(\log \left(1 / u_{n}\right)\right)^{\kappa}, \quad \mu\left(\left\{u_{n}\right\}\right)=u_{n}^{-\beta}
$$


for some $0<u_{0}<e^{-1}, 1<\beta<2$ and $\kappa>2 /(2-\beta)$. Then

$$
\nu([-\varepsilon, \varepsilon])=\sum_{u_{n} \leq \varepsilon} u_{n}^{2-\beta} \sim u_{n(\varepsilon)}^{2-\beta}
$$

with

$$
n(\varepsilon)=\min \left\{n ; u_{n} \leq \varepsilon\right\} .
$$

One easily checks that

$$
(\log (1 / \varepsilon))^{-\kappa} \varepsilon \leq u_{n(\varepsilon)} \leq \varepsilon,
$$

so (3.8) is satisfied. However, by studying the behaviour of $p\left(t_{n}, 0\right)$ on the sequence of times

$$
t_{n}=1 /\left(2 \mu \left(\left[u_{n},+\infty[)\right),\right.\right.
$$

we are going to prove that (4.3) does not hold. Notice that $t_{n} \sim u_{n}^{\beta} / 2$. Let us decompose $X_{t_{n}}$ into $Y_{n}^{1}+Y_{n}^{2}$ with

$$
Y_{n}^{1}=\sum_{s \leq t_{n}} \Delta X_{s} 1_{\left\{\Delta X_{s} \geq u_{n}\right\}}-t_{n} \int_{\left\{x \geq u_{n}\right\}} x d \mu(x) .
$$

Then

$$
\begin{aligned}
\operatorname{var} Y_{n}^{2}=t_{n} \nu\left(\left[0, u_{n+1}\right]\right) \sim t_{n} u_{n+1}^{2-\beta} & \sim \frac{u_{n}^{\beta}}{2}\left(\frac{u_{n}}{\left|\log u_{n}\right|^{\kappa}}\right)^{2-\beta} \\
& =\frac{u_{n}^{2}}{2}\left|\log u_{n}\right|^{-\kappa(2-\beta)} \\
& =\frac{u_{n+1}^{2}}{2}\left|\log u_{n}\right|^{\beta \kappa} .
\end{aligned}
$$

Moreover, the jumps contained in $Y_{n}^{2}$ are at most $u_{n+1}$ which is therefore smaller than the standard deviation of $Y_{n}^{2}$, so, by applying Lemma 2.7 to $Y_{n}^{2} /\left(\operatorname{var} Y_{n}^{2}\right)^{1 / 2}$ which has mean 0 and bounded jumps, we obtain that for any fixed $\varepsilon$

$$
\begin{aligned}
\mathbb{P}\left[\left|Y_{n}^{2}\right| \geq \varepsilon u_{n}\right] & \leq C \exp \left(-\varepsilon u_{n} /\left(\operatorname{var} Y_{n}^{2}\right)^{1 / 2}\right) \\
& \leq C \exp \left(-\varepsilon \sqrt{2}\left|\log u_{n}\right|^{\kappa(2-\beta) / 2}(1-o(1))\right) \\
& =o\left(u_{n}^{j}\right)=o\left(t_{n}^{j / \beta}\right)
\end{aligned}
$$

for any $j$ since $\kappa(2-\beta) / 2>1$. On the other hand, the compensator of the jumps of $Y_{n}^{1}$ in (4.4) satisfies

$$
t_{n} \int_{\left\{x \geq u_{n}\right\}} x d \mu(x) \sim t_{n} u_{n}^{1-\beta} \sim t_{n} u_{n} \mu\left(\left[u_{n}, \infty[)=u_{n} / 2,\right.\right.
$$

and the jump part is either 0 , either at least $u_{n}$, so $\left|Y_{n}^{1}\right| \geq u_{n} / 3$ almost surely for $n$ large enough. From (4.5) applied with $\varepsilon=1 / 12$, we deduce that

$$
\mathbb{P}\left[\left|X_{t_{n}}\right| \leq u_{n} / 4\right]=o\left(t_{n}^{j}\right)
$$


for any $j$. We also know from Theorem 3.1 that

$$
\limsup _{t \rightarrow 0} \sup _{x} \log \left|p^{\prime}(t, x)\right| / \log (1 / t) \leq 2 / \beta,
$$

so $p^{\prime}\left(t_{n}, x\right)$ is uniformly dominated by $t_{n}^{-\alpha}$ for $\alpha>2 / \beta$, and we deduce that

$$
\sup _{|x| \leq u_{n} / 4} p\left(t_{n}, x\right)=o\left(t_{n}^{j}\right)
$$

for any $j$. In particular, for $x=0$, we obtain that

$$
\liminf _{t \rightarrow 0}(\log p(t, 0) / \log (1 / t))=-\infty
$$

The reason for this behaviour is that the limit of renormalized $X_{t_{n}}$ is a Poisson variable and is therefore not absolutely continuous.

\section{Estimation at $\Delta$-ACCEssible points}

We now want to estimate $p(t, x)$ at fixed points $x \neq 0$. Before considering the density itself, we give a large deviation principle for the law of $X_{t}$; this principle involves the rate function

$$
M(x)=\inf \left\{n ; x \in \operatorname{supp} \nu^{(n)}\right\}
$$

which is the minimal number of jumps which is necessary to reach $x$ (the measure $\nu^{(n)}$ is the convolution of $\nu$ with itself $n$ times); for instance, in the dyadic example (4.2), $M(x)$ is the number of 1 's in the dyadic expansion of $x$ if $x$ is a non negative dyadic number, and is $+\infty$ otherwise. Let $M(B)=$ $\inf _{B} M$ be the minimal number of jumps necessary to reach $B$.

Proposition 5.1. Suppose that the support of $\mu$ is bounded, so that the level sets $\{M(x) \leq n\}$ are compact. Let $B$ be a Borel set; there exists a $C>0$ such that

$$
-M\left(B^{\circ}\right) \log (1 / t)-C \leq \log \mathbb{P}\left[X_{t} \in B\right] \leq-M(\bar{B}) \log (1 / t)+C
$$

for t small.

REMARK 5.2. This is a large deviation principle for $X_{t}$ as $t \downarrow 0$; one can look at other asymptotics; for instance, if exponential moments exist, the behaviour of $X_{n} / n$ as $n \uparrow \infty$ is given by the classical Cramér theorem, and this has been extended to more general Markov processes in Chapter 5 of Freidlin and Wentzell (1984).

Proof of the lower bound. Suppose that $B$ is open. Let $x \in B$ and $\varepsilon>0$ such that $M(x)=M(B)$ and $[x-\varepsilon, x+\varepsilon] \subset B$. Write $X_{t}$ as the sum $X_{t}^{1}+X_{t}^{2}$ of two independent Lévy processes with respective Lévy measures $\nu$ and $\mu-\nu$, and such that $X_{t}^{1}$ is a pure jump process. The distribution of $X_{t}^{1}$ is

$$
\mathbb{P}\left[X_{t}^{1} \in .\right]=\exp (-t \nu(\mathbb{R})) \sum_{n} \frac{t^{n}}{n !} \nu^{(n)} .
$$


If we estimate this expression below by its term of order $n=M(x)$, we obtain

$$
\mathbb{P}\left[\left|X_{t}^{1}-x\right| \leq \varepsilon / 2\right] \geq c t^{M(x)} .
$$

Since the probability of $\left\{\left|X_{t}^{2}\right| \leq \varepsilon / 2\right\}$ converges to 1 , we deduce

$$
\mathbb{P}\left[X_{t} \in B\right] \geq \mathbb{P}\left[\left|X_{t}^{1}-x\right| \leq \varepsilon / 2\right] \mathbb{P}\left[\left|X_{t}^{2}\right| \leq \varepsilon / 2\right] \geq c t^{M(x)} .
$$

Proof of the upper bound. Suppose that $B$ is closed; since we have assumed that the support of $\mu$ is bounded, there exists $\varepsilon>0$ such that the $\varepsilon$-neighbourhood $B^{\varepsilon}$ of $B$ satisfies $M\left(B^{\varepsilon}\right)=M(B)$. Let $r>0$ and consider the decomposition $X_{t}=X_{t}^{1}+X_{t}^{2}$ where $X_{t}^{1}$ is the sum of jumps greater in absolute value than $r$. The mean and variance of $X_{t}^{2}$ are $O(t)$, so from Lemma 2.9, one can choose $r$ so that

$$
\mathbb{P}\left[\left|X_{t}^{2}\right| \geq \varepsilon\right] \leq C t^{M(B)} .
$$

The distribution of $X_{t}^{1}$ is

$$
\mathbb{P}\left[X_{t}^{1} \in .\right]=\exp \left(-t \mu_{r}(\mathbb{R})\right) \sum_{n} \frac{t^{n}}{n !} \mu_{r}^{(n)}
$$

where $\mu_{r}$ denotes the restriction of $\mu$ to the complement of $[-r, r]$; then $\mu_{r}^{(n)}\left(B^{c}\right)=0$ for $n<M\left(B^{c}\right)$, so

$$
\mathbb{P}\left[\left|X_{t}^{1}\right| \in B^{\epsilon}\right] \leq C t^{M(B)} .
$$

We estimate the probability of $\left\{X_{t} \in B\right\}$ by adding (5.2) and (5.4).

We now study $p(t, x)$ at $\Delta$-accessible points (points satisfying $M(x)<\infty$ ). Due to the singularity of the asymptotic behaviour, the rate function for $\log p(t, x)$ will not always be $M$. We suppose that the process does not slip (assumptions of Theorem 4.3, cases $(a b c)$ ) and explain at the end of the section how the results can be extended to the case of a non-decreasing process. Notice that some results have been obtained previously for more general multidimensional Markov processes; when $\mu$ is smooth and $M(x)=1$ (one jump is sufficient to reach $x$ ), a precise equivalent for $p(t, x)$ is given in Léandre (1987); when $\mu$ is obtained by truncating the Lévy measure of a stable process and $M(x)$ is some integer, a lower bound is given in Ishikawa (1993), and a precise equivalent is obtained in Ishikawa (1994) for $\beta<1$.

The main result of this section is Theorem 5.6 below, but we first give two lemmas.

LEMMA 5.3. Under the conditions of Theorem 4.3, cases (abc), suppose that

$$
\nu^{(n)}([x-\varepsilon, x+\varepsilon]) \geq c \varepsilon^{\gamma}
$$

as $\varepsilon \rightarrow 0$, for some $\gamma \geq 0$ and some integer $n$. Then, for small $t$,

$$
p(t, x) \geq c^{\prime} t^{n+(\gamma-1) / \beta} .
$$


Proof. Write $X_{t}=X_{t}^{1}+X_{t}^{2}$ as in the proof of the lower bound in Proposition 5.1; we estimate below the law (5.1) of $X_{t}^{1}$ by its $n$th term, and if use our assumption, we obtain that

$$
\mathbb{P}\left[X_{t}^{1} \in[x-\varepsilon, x+\varepsilon]\right] \geq c \varepsilon^{\gamma} t^{n} .
$$

On the other hand, from Theorem 4.3, the density $p_{2}$ of $X_{t}^{2}$ is bounded below by $c t^{-1 / \beta}$ on $\left[-t^{1 / \beta}, t^{1 / \beta}\right]$, so

$$
p(t, x)=\mathbb{E}\left[p_{2}\left(t, x-X_{t}^{1}\right)\right] \geq c t^{-1 / \beta} \mathbb{P}\left[\left|X_{t}^{1}-x\right| \leq t^{1 / \beta}\right]
$$

and we apply the above estimate with $\varepsilon=t^{1 / \beta}$.

REMARK 5.4. Suppose that

$$
\nu^{(n)}(d y) \geq c 1_{V}(y) d y
$$

for some neighbourhood $V$ of $x$; then Lemma 5.3 can be applied with $\gamma=1$, but we now check that the assumptions of Theorem 4.3 can be omitted in this case. By using again the decomposition of $X_{t}$ of Proposition 5.1 (proof of the lower bound), we deduce from (5.1) that the law of $X_{t}^{1}$ is bounded below by a measure with density

$$
q(t, y)=c^{\prime} t^{n} 1_{V}(y)
$$

Thus

$$
p(t, x) \geq \mathbb{E}\left[q\left(t, x-X_{t}^{2}\right)\right] \geq c^{\prime} t^{n} \mathbb{P}\left[\left(x-X_{t}^{2}\right) \in V\right]
$$

and the probability tends to 1 , so the conclusion of Lemma 5.3 holds with $\gamma=1$.

Lemma 5.5. Under the conditions of Theorem 4.3, cases (abc), consider

$$
Z_{t}=X_{t}-\sum_{s \leq t} \Delta X_{s} 1_{\left\{\left|\Delta X_{s}\right|>r\right\}}
$$

with $r=r(t)$ of order $t^{1 / \beta}$. Let $\rho>0$ and $\beta^{\prime}>\beta$. Then the density $p_{t}^{Z}$ of $Z_{t}$ satisfies

$$
\begin{gathered}
|z| \leq \rho t^{1 / \beta} \Longrightarrow p_{t}^{Z}(z) \geq c t^{-1 / \beta} \\
p_{t}^{Z}(z) \leq C t^{-1 / \beta}, \quad|z| \geq t^{1 / \beta^{\prime}} \Longrightarrow p_{t}^{Z}(z) \leq C t^{-1 / \beta} \exp \left(-c t^{-\alpha}\right)
\end{gathered}
$$

with $\alpha=1 / \beta-1 / \beta^{\prime}$.

Proof. The variable $Z_{t} / t^{1 / \beta}$ is the variable $Y_{t}$ of the proof of Theorem 4.3 without its jumps greater than $r / t^{1 / \beta}$ which is of order 1 ; in particular, its $\chi$-parameter and $\nu$-measure are bounded, and its $\nu$-measure satisfies $(2.1)$ (on both sides in case (c)); thus the first and second inequalities follow from Lemmas 2.3 and 4.1, and one can also deduce that the derivative of $p_{t}^{Z}$ is dominated by $t^{-2 / \beta}$. On the other hand, one can estimate the density with

$$
p_{t}^{Z}(z)^{2} \leq 2 \int_{[-|z|,|z|]^{c}} p_{t}^{Z}(y)\left|\left(p_{t}^{Z}\right)^{\prime}(y)\right| d y \leq C t^{-2 / \beta} \mathbb{P}\left[\left|Z_{t}\right|>z\right]
$$


From Lemma 2.7,

$$
\mathbb{P}\left[\left|Z_{t}\right|>|z|\right] \leq C \exp \left(-|z| / t^{1 / \beta}\right)
$$

so

$$
p_{t}^{Z}(z) \leq C t^{-1 / \beta} \exp \left(-|z| /\left(2 t^{1 / \beta}\right)\right) .
$$

Theorem 5.6. Suppose the conditions of Theorem 4.3, cases (abc). Consider the functions

$$
\begin{gathered}
\gamma_{n}(x)=-\liminf _{\varepsilon \rightarrow 0}\left(\log \nu^{(n)}([x-\varepsilon, x+\varepsilon]) / \log (1 / \varepsilon)\right), \\
\bar{\gamma}_{n}(x)=-\limsup _{(y, \varepsilon) \rightarrow(x, 0)}\left(\log \nu^{(n)}([y-\varepsilon, y+\varepsilon]) / \log (1 / \varepsilon)\right)
\end{gathered}
$$

and

$$
\begin{aligned}
& \Gamma(x)=\inf _{n}\left(n+\left(\gamma_{n}(x)-1\right) / \beta\right), \\
& \bar{\Gamma}(x)=\inf _{n}\left(n+\left(\bar{\gamma}_{n}(x)-1\right) / \beta\right) .
\end{aligned}
$$

Then

$$
\begin{aligned}
-\Gamma(x) & \leq \liminf _{t \rightarrow 0}(\log p(t, x) / \log (1 / t)) \\
& \leq \limsup _{t \rightarrow 0}(\log p(t, x) / \log (1 / t)) \leq-\bar{\Gamma}(x) .
\end{aligned}
$$

REMARK 5.7. One can check that the functions $\bar{\gamma}_{n}$ and $\bar{\Gamma}$ are lower semicontinuous. Notice also that

$$
\begin{gathered}
n<M(x) \Longrightarrow \gamma_{n}(x)=\bar{\gamma}_{n}(x)=+\infty, \\
n \geq M(x) \Longrightarrow 0 \leq \bar{\gamma}_{n}(x) \leq \min \left(1, \gamma_{n}(x)\right),
\end{gathered}
$$

so

$$
M(x)-1 / \beta \leq \bar{\Gamma}(x) \leq \min (\Gamma(x), M(x)) .
$$

Proof. The lower bound is easily deduced from Lemma 5.3, so let us prove the upper bound. Fix $x$; choose $\beta^{\prime}>\beta$ and a sequence $\gamma_{n}^{\prime}<\bar{\gamma}_{n}(x)$. For some $0<r<1$ (it will be chosen later), consider the decomposition of $X_{t}$ into $X_{t}^{1}+X_{t}^{2}+X_{t}^{3}$ where $X_{t}^{1}$ and $X_{t}^{2}$ are respectively the sum of jumps in $[-r, r]^{c}$ and $\left[-t^{1 / \beta}, t^{1 / \beta}\right]^{c} \cap[-r, r]$. We want to estimate

$$
\mathbb{P}\left[\left|X_{t}^{1}+X_{t}^{2}-x\right| \leq t^{1 / \beta^{\prime}}\right]=\int \mathbb{P}\left[\left|X_{t}^{1}-x+y\right| \leq t^{1 / \beta^{\prime}}\right] \mathbb{P}\left[X_{t}^{2} \in d y\right] .
$$

By applying Lemma 2.9 to $X_{t}^{2}-\mathbb{E} X_{t}^{2}$ which has variance $O(t)$ and jumps bounded by $r$,

$$
\begin{aligned}
& \mathbb{P}\left[\left|X_{t}^{1}+X_{t}^{2}-x\right| \leq t^{1 / \beta^{\prime}}\right] \\
& \quad=\int_{\left|y-\mathbb{E} X_{t}^{2}\right| \leq K r} \mathbb{P}\left[\left|X_{t}^{1}-x+y\right| \leq t^{1 / \beta^{\prime}}\right] \mathbb{P}\left[X_{t}^{2} \in d y\right]+O\left(t^{K}\right)
\end{aligned}
$$


for any integer $K$; we will choose and fix

$$
K \geq \inf \left(n+\gamma_{n}^{\prime} / \beta^{\prime}\right)
$$

Thus, for any neighbourhood $V$ of 0 , if one chooses $r$ small enough, since $\mathbb{E} X_{t}^{2}$ converges to 0 , one has

$\mathbb{P}\left[\left|X_{t}^{1}+X_{t}^{2}-x\right| \leq t^{1 / \beta^{\prime}}\right] \leq \int_{V} \mathbb{P}\left[\left|X_{t}^{1}-x+y\right| \leq t^{1 / \beta^{\prime}}\right] \mathbb{P}\left[X_{t}^{2} \in d y\right]+O\left(t^{K}\right)$

The distribution of $X_{t}^{1}$ has been given in (5.3), and for $r<1$, the measure $\mu_{r}$ is dominated by $\nu / r^{2}$, so

$$
\mathbb{P}\left[X_{t}^{1} \in .\right] \leq \sum_{n} \frac{t^{n}}{r^{2 n} n !} \nu^{(n)}=\sum_{n<K} \frac{t^{n}}{r^{2 n} n !} \nu^{(n)}+O\left(t^{K}\right) .
$$

Now choose the neighbourhood $V$ of 0 so that

$$
\nu^{(n)}\left(\left[x-y-t^{1 / \beta^{\prime}}, x-y+t^{1 / \beta^{\prime}}\right]\right) \leq t^{\gamma_{n}^{\prime} / \beta^{\prime}}
$$

for $t$ small, $y$ in $V$ and $n<K$; then, from (5.8) and (5.7),

$$
\mathbb{P}\left[\left|X_{t}^{1}-x+y\right| \leq t^{1 / \beta^{\prime}}\right] \leq C t^{\inf \left(n+\gamma_{n}^{\prime} / \beta^{\prime}\right)}
$$

for $y$ in $V$, so

$$
\mathbb{P}\left[\left|X_{t}^{1}+X_{t}^{2}-x\right| \leq t^{1 / \beta^{\prime}}\right] \leq C t^{\inf \left(n+\gamma_{n}^{\prime} / \beta^{\prime}\right)} .
$$

By estimating the density $p_{3}(t,$.$) of X_{t}^{3}$ from Lemma 5.5, we deduce that

$$
\begin{aligned}
p(t, x) & =\mathbb{E} p_{3}\left(t, x-X_{t}^{1}-X_{t}^{2}\right) \\
& \leq C \exp \left(-c t^{-\alpha}\right)+C t^{-1 / \beta} \mathbb{P}\left[\left|X_{t}^{1}+X_{t}^{2}-x\right|<t^{1 / \beta^{\prime}}\right] \\
& \leq C^{\prime} t^{\inf \left(n+\gamma_{n}^{\prime} / \beta^{\prime}\right)-1 / \beta},
\end{aligned}
$$

and therefore,

$$
\limsup _{t \rightarrow 0}(\log p(t, x) / \log (1 / t)) \leq-\inf _{n}\left(n+\gamma_{n}^{\prime} / \beta^{\prime}\right)+1 / \beta .
$$

Since this holds for any $\beta^{\prime}>\beta$ and any sequence $\gamma_{n}^{\prime}<\bar{\gamma}_{n}(x)$, we can conclude.

Examples 5.8. Suppose that

$$
\mu(d x)=f(x) d x
$$

is absolutely continuous, has a compact support $\left[\rho_{1}, \rho_{2}\right]$, and that $f$ is locally bounded below and above on $\left[\rho_{1}, \rho_{2}\right] \backslash\{0\}$. Then

$$
M(x) \leq n \Longleftrightarrow n \rho_{1} \leq x \leq n \rho_{2}
$$


and $\nu^{(n)}$ has a density which is locally bounded below and above on $\{M(x) \leq$ $n\} \backslash\{0\}$. Thus $\gamma_{n}(x)=\bar{\gamma}_{n}(x)=1$ if $n \geq M(x), x \neq 0$, so $\Gamma(x)=\bar{\Gamma}(x)=$ $M(x)$. On the other hand, if

$$
\mu=\sum \mu_{n} \delta_{x_{n}}
$$

where $x_{n}$ is bounded below by some real number and has no limit point in $\mathbb{R}^{\star}$ (as in the dyadic example (4.2)), then $\gamma_{n}(x)=\bar{\gamma}_{n}(x)=0$ if $M(x)=n$ because $\nu^{(n)}$ has a mass at $x$; thus $\Gamma(x)=\bar{\Gamma}(x)=M(x)-1 / \beta$.

REMARK 5.9. Solving the minimization problems (5.5) and (5.6) explains how the process goes from 0 to $x$, and in those two examples, it uses the path containing the minimal number of jumps. In more general cases however, this is not so simple, and, even if $x$ is in the support of $\mu$, the process may find better to reach $x$ with two jumps; for instance if

$$
\mu(d x)=\delta_{1}(d x)+d x /|x|^{1+\beta},
$$

then the functions $\gamma_{n}$ and $\bar{\gamma}_{n}$ again coincide, but $\gamma_{1}(2)=1, \gamma_{2}(2)=0$, so $\Gamma(2)=\min (1,2-1 / \beta)$; if $\beta>1$ the process goes directly from 0 to 2 , but if $\beta<1$, it makes two jumps. Similarly, for $x=3$, it makes three jumps if $\beta<1 / 2$, and one jump if $\beta>1 / 2$.

In the end of this section, we explain without detailed proof how Theorem 5.6 can be extended to the non-decreasing case (case (d) of Theorem 4.3). The process slips on the right, so in order to be at point $x$ at time $t$, it must jump to a point strictly on the left of $x$; thus in order to obtain the lower bound of Lemma 5.3, one has to assume that

$$
\nu^{(n)}([x-\varepsilon, x-\alpha \varepsilon]) \geq c \varepsilon^{\gamma}
$$

as $\varepsilon \rightarrow 0$, for some $0<\alpha<1$, some $\gamma>0$ and some integer $n$. The proof is then similar, by noticing that the lower bound on $p_{2}$ holds in this case on $\left[\alpha t^{1 / \beta}, t^{1 / \beta}\right]$. The fact that one needs a stronger assumption is not surprising, since the upper bound of Theorem 5.6 can be made smaller in this case; in the proof, one has to choose the decomposition of $X_{t}$ with $X_{t}^{1}$, $X_{t}^{2}$ and $X_{t}^{3}$ non decreasing, and it appears that one needs an upper bound on $\nu^{(n)}([y-\varepsilon, y])$ instead of $\nu^{(n)}([y-\varepsilon, y+\varepsilon])$. Thus, if one defines

$$
\begin{gathered}
\gamma_{n}^{\star}(x)=-\sup _{0<\alpha<1} \liminf _{\varepsilon \rightarrow 0}\left(\log \nu^{(n)}([x-\varepsilon, x-\alpha \varepsilon]) / \log (1 / \varepsilon)\right), \\
\bar{\gamma}_{n}^{\star}(x)=-\limsup _{(y, \varepsilon) \rightarrow(x, 0), y<x}\left(\log \nu^{(n)}([y-\varepsilon, y]) / \log (1 / \varepsilon)\right)
\end{gathered}
$$

and

$$
\Gamma^{\star}(x)=\inf _{n}\left(n+\left(\gamma_{n}^{\star}(x)-1\right) / \beta\right), \quad \bar{\Gamma}^{\star}(x)=\inf _{n}\left(n+\left(\bar{\gamma}_{n}^{\star}(x)-1\right) / \beta\right),
$$

then

$$
\begin{aligned}
-\Gamma^{\star}(x) & \leq \liminf _{t \rightarrow 0}(\log p(t, x) / \log (1 / t)) \\
& \leq \limsup _{t \rightarrow 0}(\log p(t, x) / \log (1 / t)) \leq-\bar{\Gamma}^{\star}(x) .
\end{aligned}
$$


If one considers the above examples (5.9) and (5.10) with $\operatorname{supp} \mu \subset \mathbb{R}_{+}$, then

$$
\Gamma^{\star}(x)=\bar{\Gamma}^{\star}(x)=\lim _{y \uparrow \uparrow x} M(y)
$$

in the absolutely continuous case (5.9), whereas $\Gamma^{\star}$ and $\bar{\Gamma}^{\star}$ are $+\infty$ everywhere in the discrete case (5.10) (there is no accumulation of the mass of $X_{t}$ at any fixed point $x$ ).

\section{Estimation at asymptotically $\Delta$-ACCESSible POINTS}

If $\bar{\Gamma}(x)=\infty$ (or $\bar{\Gamma}^{\star}(x)=\infty$ in the non-decreasing case) then $\log (1 / p(t, x)) \gg$ $\log (1 / t)$. The aim of this section is to make a more precise study when $x$ is in the closure of $\bigcup_{n} \operatorname{supp} \nu^{(n)}$; for instance, in the dyadic example (4.2) this concerns positive non-dyadic numbers (non-slipping case), or all positive numbers (non-decreasing case). Since the proofs are similar, we consider mainly the non-slipping case, and give as remarks the extension to the nondecreasing case. We first derive the following general lower bound.

Proposition 6.1. Assume the conditions of Theorem 4.3, cases (abc) with $\beta<2$, and suppose that $\mu\left(\mathbb{R}_{+}^{+}\right)>0$. Then for any $R>0$,

$$
\liminf _{t \rightarrow 0} \inf _{0 \leq x \leq R} \frac{\log p(t, x)}{(\log t)^{2}}>-\infty
$$

REMARK 6.2. In the non-decreasing case, one has to consider the infimum on $\left[R_{1}, R_{2}\right]$ for positive $R_{1}$ and $R_{2}$.

Remark 6.3 . We have assumed that $\beta<2$, so that $X_{t}$ has no Brownian part; in this case indeed, the condition (4.1) imposes a constraint on the tail of $\mu$ near 0 (see ( 6.1 ) below). If $\beta=2$, the result of the proposition can be extended if one assumes that this constraint is satisfied; otherwise, the set of asymptotically $\Delta$-accessible points may be more complicated, and other orders of magnitude for $\log p(t, x)$ are possible.

Proof. From (4.1) and since $\mu\left(\mathbb{R}_{+}^{+}\right)>0$, we deduce that if we fix $\eta$ small enough, we have

$$
\left.\left.\forall \rho \leq \eta \quad \int_{\left\{\eta^{2} \rho<|y| \leq \rho\right\}} y^{2} d \mu(y) \geq c \rho^{2-\beta} \quad \text { and } \quad \mu(] \eta, 1 / \eta\right]\right)>0 .
$$

Thus

$$
\mu\left\{y ; \eta^{2} \rho<|y| \leq \rho\right\} \geq c \rho^{-\beta} \geq c
$$

for $\rho \leq \eta$, and if we define

$$
\left.\left.J_{n}=\left[-\eta^{2 n-1},-\eta^{2 n+1}[\cup] \eta^{2 n+1}, \eta^{2 n-1}\right] \quad(n \geq 1), \quad J_{0}=\right] \eta, 1 / \eta\right],
$$

then $\mu\left(J_{n}\right)$ is bounded below. For any integers $K$ and $n$, there exists an interval $I(K, n) \subset J_{n}$ of length $\left|J_{n}\right| /(2 K)$ and with measure $\mu(I(K, n)) \geq$ $\mu\left(J_{n}\right) /(2 K)$. If we let $K$ be the integer value of $t^{-1 / \beta}$, we deduce the existence of intervals $I_{n}=I_{n}(t) \subset J_{n}$ satisfying

$$
\left|I_{n}\right| \leq C t^{1 / \beta} \eta^{2 n}, \quad \mu\left(I_{n}\right) \geq c t^{1 / \beta} .
$$


Let $x_{n}=x_{n}(t)$ be a point of $I_{n}$; then $\left|x_{n}\right|$ is a decreasing sequence such that $\left|x_{n+1} / x_{n}\right| \geq \eta^{4}$, and $x_{0}$ is positive. On the other hand, for any $x \in[0, R]$, there exists a sequence of non-negative integers $k_{n}=k_{n}(t, x)$ such that

$$
\left|x-\sum_{n=0}^{N} k_{n} x_{n}\right| \leq\left|x_{N}\right| \leq \eta^{2 N-1} .
$$

More precisely, the sequence $k_{n}$ can be constructed by induction as follows; if $k_{0}, \ldots, k_{n-1}$ have been chosen, the integer $k_{n}$ is chosen so that $\sum_{j \leq n} k_{j} x_{j}$ is the best approximation of $x$ lower than $x$ if $x_{n+1}>0$, greater than $x$ if $x_{n+1}<0$. Then

$$
k_{0} \leq 1+x / x_{0} \leq 1+R / \eta \quad \text { and } \quad k_{n} \leq 1+\eta^{-4} \quad(n \geq 1)
$$

If $z$ is a real number of the form

$$
z=\sum_{n=0}^{N} \sum_{j=1}^{k_{n}} y_{n j}, \quad \text { with } \quad y_{n j} \in I_{n}
$$

then

$$
|z-x| \leq\left|x-\sum_{n=0}^{N} k_{n} x_{n}\right|+\sum_{n=0}^{N} k_{n}\left|I_{n}\right| \leq \eta^{2 N-1}+C t^{1 / \beta}
$$

from the estimation (6.2) of the length of $I_{n}$ and since the series $\sum k_{n} \eta^{2 n}$ is bounded. If we choose $N=N(t)$ so that

$$
N(t)=(\log t) /(2 \beta \log \eta)+O(1)
$$

then

$$
|z-x| \leq C t^{1 / \beta} .
$$

Let us now return to our Lévy process. We are going to use the decomposition $X_{t}=X_{t}^{1}+X_{t}^{2}$ where $X_{t}^{1}$ is the sum of the jumps greater (in absolute value) than $r=\eta^{2 N+1}$ with $N=N(t)$ like above, so that $r$ is of order $t^{1 / \beta}$. If, before time $t, X$ has exactly $k_{n}$ jumps $\Delta X \in I_{n}$, and no jump $\Delta X \in[-r, r]^{c} \backslash \bigcup I_{n}$, then $z=X_{t}^{1}$ satisfies (6.3) and therefore (6.4). Thus

$$
\begin{aligned}
\mathbb{P}\left[\left|X_{t}^{1}-x\right| \leq C t^{1 / \beta}\right] & \geq \exp \left(-t \mu\left([-r, r]^{c}\right)\right) \prod_{n \leq N(t)}\left(\left(t \mu\left(I_{n}\right)\right)^{k_{n}} / k_{n} !\right) \\
& \geq c\left(c t^{K}\right)^{N(t)}
\end{aligned}
$$

for some $K$, because $t \mu\left([-r, r]^{c}\right)$ and $k_{n}$ are bounded, and from the lower bound (6.2) on $\mu\left(I_{n}\right)$. Since $N(t)$ is of order $\log (1 / t)$, we deduce that

$$
\liminf _{t \rightarrow 0} \inf _{0 \leq x \leq R} \frac{\log \mathbb{P}\left[\left|X_{t}^{1}-x\right| \leq C t^{1 / \beta}\right]}{(\log t)^{2}}>-\infty .
$$


On the other hand, from Lemma 5.5, the density $p_{2}(t,$.$) of X_{t}^{2}$ satisfies $p_{2}(t, y) \geq c t^{-1 / \beta}$ for $|y| \leq C t^{1 / \beta}$, so

$$
\log p(t, x) \geq \log \mathbb{P}\left[\left|X_{t}^{1}-x\right| \leq C t^{1 / \beta}\right]+O(\log t)
$$

and we can conclude.

In some cases, one can make more precise the method of Proposition 6.1 in order to obtain an equivalent of $\log p(t, x)$; this is possible when the lower and upper bounds of the following proposition become similar as $\beta^{\prime} \downarrow \beta$; we will apply this result to the dyadic example (4.2).

Proposition 6.4. Suppose that $\mu$ satisfies the assumptions of Theorem 4.3, cases (abc) with $\beta<2$, and that it is supported by a countable set $\left\{x_{n}, n \in \mathbb{N}\right\}$ such that $\left|x_{n+1}\right| \leq\left|x_{n}\right|$; denote by $\mu_{n}$ the mass $\mu\left(\left\{x_{n}\right\}\right)$. Suppose that

$$
N=N(t)=\max \left\{n ;\left|x_{n}\right|>t^{1 / \beta}\right\}=O(\log (1 / t)) .
$$

Let $\rho>0$ and $\beta^{\prime}>\beta$. Suppose that $\mu\left(\mathbb{R}_{+}^{*}\right)>0$ and let $x>0$. Then $\log p(t, x)$ is bounded below and above by expressions of type

$$
-\min \sum_{n=0}^{N}\left(k_{n} \log \left(1 /\left(t \mu_{n}\right)\right)+\log \left(k_{n} !\right)\right)+O(\log (1 / t) \log \log (1 / t))
$$

where the minimum is taken with respect to the sequences $\left(k_{n}\right)$ such that

$$
\left|x-\sum_{n=0}^{N} k_{n} x_{n}\right| \leq \rho t^{1 / \beta}
$$

for the lower bound, and

$$
\left|x-\sum_{n=0}^{N} k_{n} x_{n}\right| \leq t^{1 / \beta^{\prime}}
$$

for the upper bound.

REMARK 6.5. One deduces from (4.1) that for some $c_{0}>0$, the measure $\mu$ must give positive mass to all the sets $\left\{c_{0} \varepsilon \leq|x| \leq \varepsilon\right\}$ for $\varepsilon$ small enough; thus these sets must contain at least one point $x_{n}$, so that $N(t)$ is bounded below by some $c \log (1 / t)$. The assumption of the proposition therefore says that $N(t)$ is exactly of order $\log (1 / t)$.

REMARK 6.6. If $X_{t}$ is non-decreasing, these bilateral constraints have to be replaced by unilateral ones of type

$$
\rho_{1} t^{1 / \beta} \leq x-\sum k_{n} x_{n} \leq \rho_{2} t^{1 / \beta}, \quad 0 \leq x-\sum k_{n} x_{n} \leq t^{1 / \beta^{\prime}} .
$$

REMARK 6.7. The proposition can be extended to unbounded positive sequences $\left\{x_{n}, n \in \mathbb{Z}\right\}$ with $\lim _{-\infty} x_{n}=+\infty$, because jumps greater than 
$x$ can be neglected in the following analysis. However, other cases (if for instance both $+\infty$ and $-\infty$ are limit points of $x_{n}$ ) may be more delicate.

Proof. Consider the decomposition $X_{t}=X_{t}^{1}+X_{t}^{2}$ where $X_{t}^{1}$ is the sum of jumps of size $x_{n}, n \leq N(t)$. The density $p_{2}$ of $X_{t}^{2}$ can be estimated from Lemma 5.5 , so since $p(t, x)$ is the expectation of $p_{2}\left(t, x-X_{t}^{1}\right)$, we deduce

$$
\begin{aligned}
c t^{-1 / \beta} \mathbb{P}\left[\left|X_{t}^{1}-x\right| \leq \rho t^{1 / \beta}\right] & \leq p(t, x) \\
& \leq C t^{-1 / \beta} \mathbb{P}\left[\left|X_{t}^{1}-x\right| \leq t^{1 / \beta^{\prime}}\right]+\exp \left(-c t^{-\alpha}\right)
\end{aligned}
$$

for $\alpha>0$; from Proposition 6.1, the exponential can be neglected, so

$$
\begin{aligned}
\log \mathbb{P}\left[\left|X_{t}^{1}-x\right| \leq \rho t^{1 / \beta}\right] & -C \log (1 / t) \\
& \leq \log p(t, x) \\
& \leq \log \mathbb{P}\left[\left|X_{t}^{1}-x\right| \leq t^{1 / \beta^{\prime}}\right]+C \log (1 / t)
\end{aligned}
$$

We have

$$
X_{t}^{1}=\sum_{n=0}^{N} K_{n} x_{n}
$$

where $K_{n}$ is the number of jumps at $x_{n}$ before time $t$; notice that the conditions on $X_{t}^{1}$ in $(6.8)$ can be translated by saying that the sequence $K_{n}$ should satisfy (6.6) or (6.7). Moreover, $K_{n}$ is a Poisson variable with mean $t \mu_{n}$ and the sum of these means for $n \leq N$ is bounded; in particular, the expectation of $\exp K_{n}$ is bounded, so

$$
\mathbb{P}\left[K_{n} \geq N^{3}\right] \leq C e^{-N^{3}},
$$

and

$$
\mathbb{P}\left[\exists n \leq N, \quad K_{n} \geq N^{3}\right] \leq C(N+1) e^{-N^{3}} \leq \exp \left(-c(\log (1 / t))^{3}\right)
$$

is very small; thus we can add the conditions $K_{n}<N^{3}$ in the probabilities of both sides of $(6.8)$. We deduce that $\log p(t, x)$ is bounded below and above by expressions of type

$$
\log \mathbb{P}\left[\exists\left(k_{n}\right) \in \mathcal{K} \quad \forall n \leq N \quad K_{n}=k_{n}\right]+O(\log (1 / t)),
$$

where $\mathcal{K}$ is the set of sequences $\left(k_{n}\right)$ satisfying (6.6) for the lower bound, (6.7) for the upper bound, and the additional constraint $k_{n}<N^{3}$. On the other hand,

$$
\begin{aligned}
\log \mathbb{P}\left[\forall n \leq N \quad K_{n}=k_{n}\right] & =-\sum_{n=0}^{N}\left(k_{n} \log \left(1 /\left(t \mu_{n}\right)\right)+\log \left(k_{n} !\right)+t \mu_{n}\right) \\
& =-\sum_{n=0}^{N}\left(k_{n} \log \left(1 /\left(t \mu_{n}\right)\right)+\log \left(k_{n} !\right)\right)+O(1),
\end{aligned}
$$


so

$$
\begin{aligned}
\log \mathbb{P}\left[\exists\left(k_{n}\right) \in \mathcal{K}\right. & \left.\forall n \leq N \quad K_{n}=k_{n}\right] \\
& =-\min _{\mathcal{K}} \sum_{n=0}^{N}\left(k_{n} \log \left(1 /\left(t \mu_{n}\right)\right)+\log \left(k_{n} !\right)\right)+O(\log |\mathcal{K}|) .
\end{aligned}
$$

The cardinal of $\mathcal{K}$ is at most the number of sequences $k_{n}$ satisfying $k_{n}<N^{3}$, so $\log |\mathcal{K}|$ has the correct order $\log (1 / t) \log \log (1 / t)$ of $(6.5)$. We deduce that the proposition holds with the additional constraint $k_{n}<N^{3}$ in the optimization problem (6.5), and we now have to verify that we can omit it. This does not cause any problem for the upper bound (because the bound after omitting the constraint is larger). For the lower bound, let us suppose that a minimizing sequence does not satisfy $k_{n}<N^{3}$, so that $k_{j} \geq N^{3}$ for some $j \leq N$; since $t \mu_{n}$ is bounded, one has

$$
k_{n} \log \left(1 /\left(t \mu_{n}\right)\right)+\log \left(k_{n} !\right) \geq \log \left(k_{n} !\right)-C k_{n} \geq-C^{\prime}
$$

and

$$
k_{j} \log \left(1 /\left(t \mu_{j}\right)\right)+\log \left(k_{j} !\right) \geq \log \left(k_{j} !\right)-C k_{j} \gg k_{j} \geq N^{3} .
$$

Thus the value of the cost functional is at least of order $(\log (1 / t))^{3}$, so the lower bound stated in the proposition is in this case smaller than the bound of Proposition 6.1.

Example 6.8. Consider the dyadic example (4.2) (non-slipping or nondecreasing cases). Then the behaviour of $p(t, x)$ for $x>0$ will depend on the proportion of 1 's in the dyadic expansion $d_{n}=d_{n}(x)$ of $x$ given by $x=\sum_{n \in \mathbb{Z}} d_{n} 2^{-n}$. Let

$$
N=N(t)=\min \left\{n ; 2^{(n+2) \beta} \geq 1 / t\right\}
$$

so that $N(t) \sim \log (1 / t) /(\beta \log 2)$. If one solves the optimization problem

$$
\min \sum_{n \leq N} k_{n}(\log (1 / t)-n \beta \log 2)
$$

amongst sequences $\left(k_{n}\right)$ such that $\sum k_{n} 2^{-n}$ is some fixed dyadic number of order $N$, it appears that one has to choose for $k_{n}$ the dyadic coefficients of the number (verify that a jump at $2^{-n}$ is cheaper than two jumps at $2^{-n-1}$ ). Moreover, $\sum \log \left(k_{n} !\right)$ is in this case 0 and is therefore also minimal, so the optimization problem of Proposition 6.4 is solved by choosing the best (in the sense of the cost functional (6.9)) dyadic number satisfying the constraint (6.6) or $(6.7)$, and $\log p(t, x)$ is bounded below and above by expressions of type

$$
-\sum_{n \leq N} k_{n}(\log (1 / t)-n \beta \log 2)+O(\log (1 / t) \log \log (1 / t))
$$

where $k_{n}$ are the dyadic coefficients of this best dyadic number. One can notice that for the lower bound, one has to choose $\rho$ large enough in order to have at least one sequence $k_{n}$ satisfying the constraint (6.6). Notice also 
that the best coefficients $\left(k_{n}, n \leq N\right)$ depend on $t$ (since the constraints involve $t$ ); however, in many cases, only the last values will depend on $t$ and most values of $k_{n}$ will actually coincide with $d_{n}(x)$. If

$$
\lim K^{-1} \sum_{n=0}^{K} d_{n}(x)=\rho
$$

for some $0<\rho<1$ (proportion of 1 's in the dyadic expansion of $x$ ), then the sequence $d_{n}$ asymptotically does not contain too long subsequences of 0 's or of 1 's, and one can deduce that $d_{n}$ is a good suboptimal solution of the minimization problem, so that

$$
\begin{aligned}
\log p(t, x) & \sim-\log (1 / t) \sum_{n \leq N(t)} d_{n}(x)+\beta \log 2 \sum_{n \leq N(t)} n d_{n}(x) \\
& \sim-\rho N(t) \log (1 / t)+\rho \beta(\log 2) N(t)^{2} / 2 \\
& \sim \frac{-\rho}{2 \beta \log 2}(\log t)^{2} .
\end{aligned}
$$

This holds if $x$ is a rational non-dyadic number (in this case $d_{n}$ is periodic after some rank); this also holds for almost any $x$ relatively to the Lebesgue measure (in this case $\rho=1 / 2$ ). Moreover, if $x$ is a dyadic number and if $X_{t}$ is non-decreasing (apply $\S 5$ in the non slipping case), one has to reach $x$ from below, and one can check that (6.10) holds with $\rho=1$ (loosely speaking, $x$ has two expansions, and one has to consider the one where all terms are 1 after some rank).

\section{Estimation at $\Delta$-INACCESSIBle Points}

We now consider points which cannot be reached by jumping; this means that they are not in

$$
F=\operatorname{supp} \sum_{n=0}^{\infty} \nu^{(n)} .
$$

We check that the density is much smaller in this case. We first consider the case where $X_{t}$ contains a non trivial Brownian part.

Proposition 7.1. Suppose that the diffusion coefficient $\sigma$ is not zero. Then, if $\delta(x)$ denotes the distance of $x$ to $F$,

$$
\lim _{t \rightarrow 0}(t \log p(t, x))=-\delta^{2}(x) /\left(2 \sigma^{2}\right) .
$$

Proof. Write $X_{t}=\sigma W_{t}+Y_{t}$ where $W_{t}$ is a standard Wiener process. Then

$$
p(t, x)=\frac{1}{\sigma \sqrt{2 \pi t}} \mathbb{E}\left[\exp -\frac{\left(x-Y_{t}\right)^{2}}{2 \sigma^{2} t}\right] .
$$

Let us first prove the lower bound. Choose $y \in F$ such that $|y-x|=\delta(x)$. Fix $\varepsilon>0$ and let $n=n(\varepsilon)$ be the first integer such that $] y-\varepsilon, y+\varepsilon[$ intersects the support of $\nu^{(n)}$; then

$$
\mathbb{P}\left[\left|Y_{t}-y\right| \leq \varepsilon\right] \geq c_{\varepsilon} t^{n}
$$


from Proposition 5.1, and we obtain from (7.1) that

$$
p(t, x) \geq \frac{1}{\sigma \sqrt{2 \pi t}} \mathbb{P}\left[\left|Y_{t}-y\right| \leq \varepsilon\right] \exp -\frac{(\delta(x)+\varepsilon)^{2}}{2 \sigma^{2} t},
$$

so

$$
\liminf _{t \rightarrow 0} t \log p(t, x) \geq-\frac{(\delta(x)+\varepsilon)^{2}}{2 \sigma^{2}} .
$$

Since this holds for any $\varepsilon>0$, we conclude. Let us now prove the upper bound; if $x \in F$, we can apply the estimation of Theorem 3.1(a), so suppose $x \notin F$; we consider separately the two cases $\mu$ finite and $\mu$ infinite. If $\mu$ is finite, we can write $Y_{t}=t \bar{\chi}+\bar{Y}_{t}$ where $\bar{Y}_{t}$ is pure jump process with a finite number of jumps; the support of $\bar{Y}_{t}$ is exactly $F$ so

$$
p(t, x) \leq \frac{1}{\sigma \sqrt{2 \pi t}} \exp -\frac{\delta^{2}(x-t \bar{\chi})}{2 \sigma^{2} t}
$$

and we deduce the result. If $\mu$ is infinite, suppose for instance that $\mu\left(\mathbb{R}_{+}^{*}\right)$ is infinite; then $\mu\left(\mathbb{R}_{-}^{\star}\right)=0$ because otherwise $F=\mathbb{R}$. Thus $F$ is $\mathbb{R}_{+}, x$ is negative and $\delta(x)=-x$. The Laplace transform of $Y_{t}$ is given from (1.2) by

$$
\mathbb{E} \exp -\lambda Y_{t}=\exp \left(-\lambda t \chi-t \int_{1}^{\infty}\left(1-e^{-\lambda y}\right) d \mu(y)+t \psi(\lambda)\right)
$$

for $\lambda \geq 0$, with

$$
\begin{aligned}
\psi(\lambda) & =\int_{0}^{1}\left(e^{-\lambda y}+\lambda y-1\right) d \mu(y) \\
& \left.\left.=\left(e^{-\lambda}+\lambda-1\right) \nu(] 0,1\right]\right)+\int_{0}^{1}\left(\lambda y+(\lambda y+2) e^{-\lambda y}-2\right) \frac{\nu(] 0, y])}{y^{3}} d y \\
& \left.\left.=\left(e^{-\lambda}+\lambda-1\right) \nu(] 0,1\right]\right)+\lambda^{2} \int_{0}^{1}\left(y+(y+2) e^{-y}-2\right) \frac{\nu(] 0, y / \lambda])}{y^{3}} d y .
\end{aligned}
$$

In particular, $\psi(\lambda) \ll \lambda^{2}$ as $\lambda \rightarrow+\infty$. We also check that $\psi$ is strictly increasing from $\mathbb{R}_{+}$onto itself, so if $\psi^{-1}$ denotes the inverse function, $\psi^{-1}(\rho) \gg$ $\sqrt{\rho}$ as $\rho \rightarrow+\infty$. By taking $\lambda=\psi^{-1}\left(1 / t^{2}\right)$, we obtain that for any $0<\varepsilon<$ $|x|$,

$\log \mathbb{P}\left[Y_{t} \leq-\varepsilon\right] \leq-\lambda \varepsilon+\log \mathbb{E} \exp -\lambda Y_{t} \leq-\lambda(\varepsilon+t \chi)+1 / t+O(t) \ll-1 / t$ because $\lambda \gg 1 / t$. From (7.1),

$$
p(t, x) \leq \frac{1}{\sigma \sqrt{2 \pi t}}\left(\mathbb{P}\left[Y_{t} \leq-\varepsilon\right]+\exp \left(-(\delta(x)-\varepsilon)^{2} /\left(2 \sigma^{2} t\right)\right)\right),
$$

so

$$
\limsup _{t \rightarrow 0} t \log p(t, x) \leq-(\delta(x)-\varepsilon)^{2} /\left(2 \sigma^{2}\right)
$$

and one only has to let $\varepsilon \downarrow 0$. 
By letting $\sigma \rightarrow 0$ in previous result, it seems that $t \log p(t, x) \rightarrow-\infty$ at $\Delta$-inaccessible points when there is no Brownian part. The next result gives a precise statement.

Theorem 7.2. Assume the conditions of Theorem 4.3 with $1<\beta<2$, and suppose that $\mu\left(\mathbb{R}_{-}^{\star}\right)=0$. Fix positive $T$ and $\alpha$ and consider the function $p(t, x)$ on the domain $D=] 0, T] \times]-\infty,-\alpha]$. Let $D \cup\{\partial\}$ be the one-point compactification of $D$. Consider the function

$$
h(\lambda)=\log \mathbb{E} \exp -\lambda X_{1}
$$

on $\mathbb{R}_{+}$, and its Legendre transform

$$
\Lambda(y)=\sup _{\lambda \geq 0}(\lambda y-h(\lambda))
$$

Then

$$
\log (1 / p(t, x)) \sim t \Lambda(-x / t)
$$

as $(t, x) \rightarrow \partial ;$ moreover

$$
c\left(|x|^{\beta / t}\right)^{1 /(\beta-1)} \leq \log (1 / p(t, x)) \leq C\left(|x|^{\beta} / t\right)^{1 /(\beta-1)} .
$$

Notice that the theorem gives simultaneously the asymptotic behaviours for $t \rightarrow 0$ and $x \rightarrow-\infty$; this is due to the fact that these behaviours depend only on the tail of $\mu$ at 0 , and that we have assumed an approximate scaling property on this tail. One can also prove a large deviation principle for the law of $X_{t}$ restricted to $\mathbb{R}_{-}$, and this principle involves the same rate function $\Lambda$; this comes from the regularity of $\Lambda$; if one compares with $\Delta$-accessible points, the situation was different because the rate functions were singular in the case of a singular Lévy measure. Notice also that $\Lambda$ is the rate function of the classical Cramér theorem which deals with the asymptotic behaviour of $X_{n} / n$; actually, in the case of a stable process, the behaviours of $X_{t}$ and $X_{n} / n$ can be deduced from each other (see the discussion at the end of the section). The basic tool of the proof will be a change of probability. We first prove some lemmas.

LEMMA 7.3. The function $H(\lambda)=h^{\prime}(\lambda)$ is strictly increasing from $\mathbb{R}_{+}$onto $\left[H(0),+\infty\left[\right.\right.$ and is of order $\lambda^{\beta-1}$ as $\lambda \rightarrow+\infty$; the inverse function $H^{-1}(y)$ and the function $\Lambda(y)$ are respectively of order $y^{1 /(\beta-1)}$ and $y^{\beta /(\beta-1)}$ as $y \rightarrow+\infty$.

Proof. The function $h$ is given from (1.2) by

$$
h(\lambda)=-\lambda \chi-\int_{1}^{\infty}\left(1-e^{-\lambda y}\right) d \mu(y)+\int_{0}^{1}\left(e^{-\lambda y}+\lambda y-1\right) d \mu(y) .
$$

It is strictly convex, and

$$
H(\lambda)=-\chi-\int_{1}^{\infty} y e^{-\lambda y} d \mu(y)+\phi(\lambda)
$$


with

$$
\begin{aligned}
\phi(\lambda) & =\int_{0}^{1}\left(1-e^{-\lambda y}\right) y d \mu(y) \\
& =\left(1-e^{-\lambda}\right) \nu([0,1])+\int_{0}^{1}\left(1-e^{-\lambda y}-\lambda y e^{-\lambda y}\right) \frac{\nu([0, y])}{y^{2}} d y .
\end{aligned}
$$

Since $\nu([0, y])$ is of order $y^{2-\beta}$ as $y \rightarrow 0$ we deduce that $\phi(\lambda)$ is order $\lambda^{\beta-1}$ as $\lambda \rightarrow \infty$, so $H(\lambda)$ is also of order $\lambda^{\beta-1}$, and $H^{-1}(y)$ is of order $y^{1 /(\beta-1)}$. By solving the optimization problem (7.2), one has

$$
\Lambda(y)=y H^{-1}(y)-\left(h \circ H^{-1}\right)(y)
$$

for $y>H(0)$, so $\Lambda^{\prime}=H^{-1}$ and the order of $\Lambda(y)$ can be deduced from the order of $H^{-1}(y)$ by an integration.

Lemma 7.4. For $\lambda>0$, consider the process

$$
L_{t}=\exp \left(-\lambda X_{t}-t h(\lambda)\right)
$$

Then $L_{t}$ is a martingale, and if $\widetilde{\mathbb{P}}$ is the probability measure with density $L_{t}$ with respect to $\mathbb{P}$ on $\sigma\left(X_{s}, s \leq t\right)$, then $X_{t}$ is under $\widetilde{\mathbb{P}}$ a Lévy process with parameters

$$
\tilde{\chi}=\chi-\phi(\lambda), \quad d \tilde{\mu}(y)=e^{-\lambda y} d \mu(y),
$$

where the function $\phi$ was defined in (7.6).

Proof. The process $\log L_{t}$ is a Lévy process, and if $s \leq t$, it follows from the value of the Laplace transform of $X_{t}-X_{s}$ that the mean of $L_{t} / L_{s}$ is 1. Thus $L_{t}$ is a martingale, and it is also easy to verify that $X_{t}$ is under $\widetilde{\mathbb{P}}$ a Lévy process. We have to compute its parameters; for any real $w$, from (1.2) and (7.4),

$$
\begin{aligned}
\widetilde{\mathbb{E}}\left[e^{i w X_{t}}\right]=\mathbb{E}\left[e^{(i w-\lambda) X_{t}}\right] \exp (-t h(\lambda)) \\
=\exp \left((i w-\lambda) t \chi+t \int_{0}^{1}\left(e^{(i w-\lambda) y}-1-(i w-\lambda) y\right) d \mu(y)\right. \\
\quad-t \int_{1}^{\infty}\left(1-e^{(i w-\lambda) y}\right) d \mu(y)+\lambda t \chi \\
\left.\quad-t \int_{0}^{1}\left(e^{-\lambda y}+\lambda y-1\right) d \mu(y)+t \int_{1}^{\infty}\left(1-e^{-\lambda y}\right) d \mu(y)\right) \\
=\exp \left(i w t \chi+t \int_{0}^{1}\left(e^{i w y}-1-i w y e^{\lambda y}\right) e^{-\lambda y} d \mu(y)\right. \\
\left.\quad-t \int_{1}^{\infty}\left(1-e^{i w y}\right) e^{-\lambda y} d \mu(y)\right) .
\end{aligned}
$$

We now identify the parameters with the Lévy-Khintchine formula (1.1).

Lemma 7.5. Consider the variable $L_{t}$ defined by (7.8) with $\lambda=\lambda(t, x)=$ $H^{-1}(-x / t)$. We denote by $\widetilde{\mathbb{P}}_{t, x}$ the probability on $\sigma\left(X_{s}, s \leq t\right)$ admitting 
the density $L_{t}$ with respect to $\mathbb{P}$. If $\tilde{p}(t,$.$) is the continuous density of X_{t}$ under $\widetilde{\mathbb{P}}_{t, x}$, then $\tilde{p}(t, x)$ is of order $\left(|x|^{2-\beta} / t\right)^{1 /(2 \beta-2)}$ as $(t, x) \rightarrow \partial$.

Proof. We deduce from Lemma 7.4, (7.5) and $H(\lambda)=-x / t$ that under $\widetilde{\mathbb{P}}_{t, x}$, the variable $X_{t}$ has parameters

$$
\chi_{t, x}=t \chi-t \phi(\lambda)=x-t \int_{1}^{\infty} y e^{-\lambda y} d \mu(y)
$$

and

$$
\mu_{t, x}(d y)=t \exp (-\lambda y) \mu(d y)
$$

We deduce that the parameters of the variable $Y_{t}=\left(|x|^{2-\beta} / t\right)^{1 /(2 \beta-2)}\left(X_{t}-\right.$ $x$ ) are given by

$$
\begin{gathered}
\chi_{t, x}^{Y}=-t\left(|x|^{2-\beta} / t\right)^{1 /(2 \beta-2)} \int_{\left(|x|^{2-\beta} / t\right)^{-1 /(2 \beta-2)}}^{\infty} y e^{-\lambda y} d \mu(y), \\
\nu_{t, x}^{Y}([0, \varepsilon])=t \int_{0}^{\varepsilon\left(|x|^{2-\beta} / t\right)^{-1 /(2 \beta-2)}}\left(\left(|x|^{2-\beta} / t\right)^{1 /(\beta-1)} y^{2} \wedge 1\right) e^{-\lambda y} d \mu(y) .
\end{gathered}
$$

From Lemma 7.3, we have

$$
c(|x| / t)^{1 /(\beta-1)} \leq \lambda \leq C(|x| / t)^{1 /(\beta-1)},
$$

so

$$
\begin{aligned}
\left|\chi_{t, x}^{Y}\right| \leq t\left(|x|^{2-\beta} / t\right)^{1 /(2 \beta-2)} \exp \left(-c\left(|x|^{\beta} / t\right)^{1 /(2 \beta-2)}\right) & \\
& \int_{\left(|x|^{2-\beta} / t\right)^{-1 /(2 \beta-2)}}^{\infty} y e^{-c y} d \mu(y) \\
\leq & \exp \left(-c^{\prime}\left(|x|^{\beta} / t\right)^{1 /(2 \beta-2)}\right)
\end{aligned}
$$

is very small; for the $\nu$-measure, if $|x|^{2-\beta} / t \geq 1$, one gets

$$
\begin{aligned}
\nu_{t, x}^{Y}\left(\mathbb{R}_{+}\right) & \leq t\left(|x|^{2-\beta} / t\right)^{1 /(\beta-1)} \int_{0}^{\infty} e^{-\lambda y} d \nu(y) \\
& =(|x| / t)^{(2-\beta) /(\beta-1)} \lambda \int_{0}^{\infty} e^{-\lambda y} \nu([0, y]) d y \\
& \leq C(|x| / t)^{(2-\beta) /(\beta-1)} \lambda^{\beta-2}
\end{aligned}
$$


which is bounded from (7.9). Moreover, for $0<\varepsilon<1$,

$$
\begin{aligned}
& \nu_{t, x}^{Y}([0, \varepsilon])=\left(\frac{|x|}{t}\right)^{(2-\beta) /(\beta-1)} \int_{0}^{\varepsilon\left(|x|^{2-\beta / t)^{-1 /(2 \beta-2)}}\right.} e^{-\lambda y} d \nu(y) \\
& =\left(\frac{|x|}{t}\right)^{(2-\beta) /(\beta-1)} \\
& \left(\exp \left(-\lambda \varepsilon\left(\frac{|x|^{2-\beta}}{t}\right)^{-1 /(2 \beta-2)}\right) \nu\left(\left[0, \varepsilon\left(|x|^{2-\beta} / t\right)^{-1 /(2 \beta-2)}\right]\right)\right. \\
& \left.+\lambda \int_{0}^{\varepsilon\left(|x|^{2-\beta} / t\right)^{-1 /(2 \beta-2)}} e^{-\lambda y} \nu([0, y]) d y\right) \\
& \geq c\left(\frac{|x|}{t}\right)^{(2-\beta) /(\beta-1)} \\
& \left(\exp \left(-C \varepsilon\left(\frac{|x|^{\beta}}{t}\right)^{1 /(2 \beta-2)}\right)\left(|x|^{2-\beta} / t\right)^{-(2-\beta) /(2 \beta-2)} \varepsilon^{2-\beta}\right. \\
& \left.+(|x| / t)^{-(2-\beta) /(\beta-1)} \int_{0}^{c \varepsilon\left(|x|^{\beta} / t\right)^{1 /(2 \beta-2)}} e^{-y} y^{2-\beta} d y\right) \\
& \geq c\left(\exp \left(-C \varepsilon\left(|x|^{\beta} / t\right)^{1 /(2 \beta-2)}\right)\left(|x|^{\beta} / t\right)^{(2-\beta) /(2 \beta-2)} \varepsilon^{2-\beta}\right. \\
& \left.+\int_{0}^{c \varepsilon\left(|x|^{\beta} / t\right)^{1 /(2 \beta-2)}} e^{-y} y^{2-\beta} d y\right)
\end{aligned}
$$

where we have used the estimation (7.9) on $\lambda$ and the lower bound on the tail of $\nu$ at 0 . When $\varepsilon \leq\left(|x|^{\beta} / t\right)^{-1 /(2 \beta-2)}$, the first term is bounded below by some $c \varepsilon^{2-\beta}$, and otherwise, the second term is bounded below by a positive constant number. We deduce that $\nu_{t, x}^{Y}$ satisfies the assumptions of lemmas 2.3 and 4.1, so that the density of $Y_{t}$ at 0 is bounded below and above by positive constant numbers.

Proof of Theorem 7.2. From (7.7), one has for $\lambda=H^{-1}(-x / t)$ that

$$
\operatorname{th}(\lambda)=-\lambda x-t \Lambda(-x / t)
$$

so the density $L_{t}$ of $\widetilde{\mathbb{P}}_{t, x}$ with respect to $\mathbb{P}$ satisfies

$$
L_{t}=\exp \left(-\lambda\left(X_{t}-x\right)+t \Lambda(-x / t)\right)
$$

Thus the density $\widetilde{p}(t,$.$) of X_{t}$ under $\widetilde{\mathbb{P}}_{t, x}$ is given by

$$
\widetilde{p}(t, z)=p(t, z) \mathbb{E}\left[L_{t} \mid X_{t}=z\right]=p(t, z) \exp (-\lambda(z-x)+t \Lambda(-x / t))
$$

for almost any $z$. Since both sides are continuous in $z$, we can take the value at $z=x$ and from Lemma 7.5 we obtain

$$
\log p(t, x)=-t \Lambda(-x / t)+\log \tilde{p}(t, x)=-t \Lambda(-x / t)+O(\log (|x| / t)) .
$$

The estimation (7.3) is then deduced from Lemma 7.3. 
Example 7.6. Suppose that $h(\lambda)=\lambda^{\beta}$, so that $X_{t}$ is a completely asymmetric stable process. Then

$$
\Lambda(y)=(\beta-1)(y / \beta)^{\beta /(\beta-1)},
$$

so

$$
\log p(t, x) \sim-\frac{\beta-1}{\beta^{\beta /(\beta-1)}}\left(\frac{|x|^{\beta}}{t}\right)^{1 /(\beta-1)} .
$$

Write this result at time $t=n^{1-\beta}$ for $n$ a large integer; from the scaling property, this gives the law of $X_{n} / n$, and the right-hand side of (7.10) is $-n \Lambda(-x)$; we recognize the rate function of the classical Cramér theorem. If now $\mu$ has a bounded support, the behaviour of $X_{n} / n$ cannot be deduced from Theorem 7.2 , but one can apply the Cramér theorem and get a large deviation principle with rate function $\Lambda$ on both $\mathbb{R}_{+}^{+}$and $\mathbb{R}_{-}^{\star}$; this can be extended to more general Markov processes (see Chapter 5 of Freidlin and Wentzell (1984) for the large deviation principle, and Ishikawa (1995) for an estimation of the density).

\section{REFERENCES}

Bichteler, K., Gravereaux, J. B. and Jacod, J. (1987), Malliavin calculus for processes with jumps, Stochastics Monographs 2, Gordon and Breach.

Bismut, J. M. (1983), Calcul des variations stochastique et processus de sauts, $Z$. Wahrscheinlichkeitstheorie verw. Gebiete 63 147-235.

Freidlin, M. I. and Wentzell, A. D. (1984), Random perturbations of dynamical systems, Springer.

IsHIKAWA, Y. (1993), On the lower bound of the density for jump processes in small time, Bull. Sc. Math., $2^{e}$ série 117 463-483.

Ishikawa, Y. (1994), Asymptotic behavior of the transition density for jump type processes in small time, Tôhoku Math. J. 46 443-456.

IsHIKAWA, Y. (1995), Large deviation estimate of transition densities for jump processes, preprint.

LÉandre, R. (1987), Densité en temps petit d'un processus de sauts, in: Séminaire de Probabilités XXI, Lect. N. in Math. 1247, Springer.

Picard, J. (1996), On the existence of smooth densities for jump processes, Probab. Theory Relat. Fields 105 481-511.

Rubin, H. (1967), Supports of convolutions of identical distributions, in: Proc. 5th Berkeley Symposium, Vol. II, Part 1, Univ. Calif. Press.

Tucker, H. G. (1962), Absolute continuity of infinitely divisible distributions, Pacific J. Math. 12 1125-1129.

TUCKER, H. G. (1965), On a necessary and sufficient condition that an infinitely divisible distribution be absolutely continuous, Transactions A.M.S. 118 316-330.

Yamazato, M. (1994), Absolute continuity of transition probabilities of multidimensional processes with stationary independent increments, Theory Prob. 39 347-354.

Laboratoire de Mathématiques Appliquées, URA 1501 du CNRS, Université Blaise Pascal, 63177 Aubière Cedex, France.

E-MAIL: picard@ucfma.univ-bpclermont.fr 